\title{
TWISTED SUPPORT VARIETIES
}

\author{
PETTER ANDREAS BERGH
}

\begin{abstract}
We define and study twisted support varieties for modules over an Artin algebra, where the twist is induced by an automorphism of the algebra. Under a certain finite generation hypothesis we show that the twisted variety of a module satisfies Dade's Lemma and is one dimensional precisely when the module is periodic with respect to the twisting automorphism. As a special case we obtain results on $D$ Tr-periodic modules over Frobenius algebras.
\end{abstract}

\section{Introduction}

In this paper we define and study a new type of cohomological support varieties, called twisted support varieties, for modules over Artin algebras, varieties sharing many of the properties of those defined for Artin algebras in [EHSST] and [SnS]. In those papers the underlying geometric object used to define support varieties was the Hochschild cohomology ring of an algebra, whereas our geometric object is a "twisted" Hochschild cohomology ring, the twist being induced by an automorphism of the algebra.

By introducing a finite generation hypothesis similar to the one used in Be1] we are able to relate the dimension of the twisted variety of a module to the polynomial growth of the lengths of the modules in its minimal projective resolution. In particular Dade's Lemma holds, that is, the twisted variety of a module is trivial if and only if the module has finite projective dimension. This property is highly desirable in any cohomological variety theory. Moreover, the modules whose twisted varieties are one dimensional are precisely those which are periodic with respect to the twisting automorphism, a concept defined in Section 4. As a special case we obtain results on $D$ Tr-periodic modules over Frobenius algebras.

Both when studying the twisted Hochschild cohomology ring and twisted support varieties we illustrate the theory with examples taken from the well known representation theory of the four dimensional Frobenius algebra

$$
k\langle x, y\rangle /\left(x^{2}, x y+q y x, y^{2}\right),
$$

where $k$ is a field and $q \in k$ is not a root of unity. Indeed, detecting the $D$ Tr-periodic modules over this algebra by means of support varieties was the motivation for this paper in the first place.

\section{Preliminaries}

Throughout we let $k$ be a commutative Artin ring and $\Lambda$ an indecomposable Artin $k$-projective $k$-algebra with Jacobson radical $\mathfrak{r}$. Unless otherwise specified all modules considered are finitely generated left modules. We denote by $\bmod \Lambda$ the category of all finitely generated left $\Lambda$-modules, and we fix a nonzero module $M \in \bmod \Lambda$ with minimal projective resolution

$$
\mathbb{P}: \cdots \rightarrow P_{2} \stackrel{d_{2}}{\longrightarrow} P_{1} \stackrel{d_{1}}{\longrightarrow} P_{0} \stackrel{d_{0}}{\longrightarrow} M \rightarrow 0 .
$$

2000 Mathematics Subject Classification. 16E05, 16E30, 16E40, 16P90.

Key words and phrases. Support varieties, twisting automorphism. 
Let $F: \bmod \Lambda \rightarrow \bmod \Lambda$ be an exact $k$-functor. It follows from a theorem of Watts (see [Rot, Corollary 3.34]) that there exists a bimodule $Q$ having the property that $F$ is naturally equivalent to the functor $Q \otimes_{\Lambda}-$, and that $Q$ may be chosen to be $F(\Lambda)$. Since $Q$ is in $\bmod \Lambda$ it has finite $k$-length and is therefore finitely generated also as a right module, and this implies that $Q$ is projective as a right module (it is flat). Suppose $Q$ is projective also as a left module (but not necessarily as a bimodule). Then if $P$ is a projective $\Lambda$-module the functor $\operatorname{Hom}_{\Lambda}(F(P),-)$, being naturally isomorphic to $\operatorname{Hom}_{\Lambda}\left(P, \operatorname{Hom}_{\Lambda}(Q,-)\right)$, is exact, hence $F(P)$ is projective.

For such a functor $F$ and a positive integer $t \in \mathbb{N}$, define a homogeneous product in the graded $k$-module

$$
\operatorname{Ext}_{\Lambda}^{t *}\left(F^{*}(M), M\right)=\bigoplus_{n=0}^{\infty} \operatorname{Ext}_{\Lambda}^{t n}\left(F^{n}(M), M\right)
$$

as follows; if $\eta$ and $\theta$ are two homogeneous elements in $\operatorname{Ext}_{\Lambda}^{t *}\left(F^{*}(M), M\right)$, then

$$
\eta \theta \stackrel{\text { def }}{=} \eta \circ F^{|\eta| / t}(\theta),
$$

where "o" denotes the Yoneda product. In other words, if $\eta$ and $\theta$ are given as exact sequences

$$
\begin{array}{r}
\eta: 0 \rightarrow M \rightarrow A_{t n-1} \rightarrow \cdots \rightarrow A_{0} \rightarrow F^{n}(M) \rightarrow 0 \\
\theta: 0 \rightarrow M \rightarrow B_{t m-1} \rightarrow \cdots \rightarrow B_{0} \rightarrow F^{m}(M) \rightarrow 0,
\end{array}
$$

then $\eta \theta$ is given as the exact sequence obtained from the Yoneda product of the sequence $\eta$ with the sequence

$$
F^{n}(\theta): 0 \rightarrow F^{n}(M) \rightarrow F^{n}\left(B_{t m-1}\right) \rightarrow \cdots \rightarrow F^{n}\left(B_{0}\right) \rightarrow F^{m+n}(M) \rightarrow 0 .
$$

Furthermore, for a $\Lambda$-module $N$, define a homogeneous right scalar action on $\operatorname{Ext}_{\Lambda}^{t *}\left(F^{*}(M), N\right)$ from $\operatorname{Ext}_{\Lambda}^{t *}\left(F^{*}(M), M\right)$ as follows; if $\mu \in \operatorname{Ext}_{\Lambda}^{t *}\left(F^{*}(M), N\right)$ and $\eta \in \operatorname{Ext}_{\Lambda}^{t *}\left(F^{*}(M), M\right)$ are homogeneous elements, then $\mu \eta \stackrel{\text { def }}{=} \mu \circ F^{|\mu| / t}(\eta)$.

Lemma 2.1. Extending the homogeneous product and scalar product defined above to all graded elements makes $\operatorname{Ext}_{\Lambda}^{t *}\left(F^{*}(M), M\right)$ into a graded $k$-algebra and $\operatorname{Ext}_{\Lambda}^{t *}\left(F^{*}(M), N\right)$ into a graded right $\operatorname{Ext}_{\Lambda}^{t *}\left(F^{*}(M), M\right)$-module.

Proof. The product in $\operatorname{Ext}_{\Lambda}^{t *}\left(F^{*}(M), M\right)$ is associative, and the right distributive law holds; if $\theta_{1}, \theta_{2}$ and $\eta$ are homogeneous elements such that $\theta_{1}$ and $\theta_{2}$ are of the same degree $t n$, then

$$
\left(\theta_{1}+\theta_{2}\right) \eta=\left(\theta_{1}+\theta_{2}\right) \circ F^{n}(\eta)=\theta_{1} \circ F^{n}(\eta)+\theta_{2} \circ F^{n}(\eta)=\theta_{1} \eta+\theta_{2} \eta .
$$

Now suppose the degree of $\eta$ is $t m$. We can represent $\theta_{i}$ as a map

$$
\Omega_{\Lambda}^{t n}\left(F^{n}(M)\right) \stackrel{f_{\theta_{i}}}{\longrightarrow} M,
$$

and since the functor $F^{m}$ preserves direct limits (in particular pushouts) and projective resolutions (though it may not preserve minimal resolutions), we see that the map

$$
F^{m}\left(\Omega_{\Lambda}^{t n}\left(F^{n}(M)\right)\right) \stackrel{F^{m}\left(f_{\theta_{i}}\right)}{\longrightarrow} F^{m}(M)
$$

represents $F^{m}\left(\theta_{i}\right)$. As $F^{m}$ is additive we have $F^{m}\left(f_{\theta_{1}}+f_{\theta_{2}}\right)=F^{m}\left(f_{\theta_{1}}\right)+F^{m}\left(f_{\theta_{2}}\right)$ as elements of $\operatorname{Hom}_{\Lambda}\left(F^{m}\left(\Omega_{\Lambda}^{t n}\left(F^{n}(M)\right)\right), F^{m}(M)\right)$, and this implies that $F^{m}\left(\theta_{1}+\theta_{2}\right)=$ $F^{m}\left(\theta_{1}\right)+F^{m}\left(\theta_{2}\right)$. Therefore the left distributive law also holds for the homogeneous product;

$$
\begin{aligned}
\eta\left(\theta_{1}+\theta_{2}\right) & =\eta \circ\left(F^{m}\left(\theta_{1}+\theta_{2}\right)\right)=\eta \circ\left(F^{m}\left(\theta_{1}\right)+F^{m}\left(\theta_{2}\right)\right) \\
& =\eta \circ F^{m}\left(\theta_{1}\right)+\eta \circ F^{m}\left(\theta_{2}\right)=\eta \theta_{1}+\eta \theta_{2} .
\end{aligned}
$$


Extending this homogeneous product in the natural way we see that $\operatorname{Ext}_{\Lambda}^{t *}\left(F^{*}(M), M\right)$ becomes a graded $k$-algebra, and similar arguments show that $\operatorname{Ext}_{\Lambda}^{t *}\left(F^{*}(M), N\right)$ is a graded right $\operatorname{Ext}_{\Lambda}^{t *}\left(F^{*}(M), M\right)$-module.

Example. Suppose $k$ is a field and $\Lambda$ is selfinjective, and consider a bimodule $B$ in the stable category $\underline{\bmod } \Lambda^{\mathrm{e}}$ of finitely generated $\Lambda^{\mathrm{e}}$-modules modulo projectives. The Auslander-Reiten translation $\tau_{\Lambda^{\mathrm{e}}}=D \operatorname{Tr}$ is a self-equivalence on $\underline{\bmod } \Lambda^{\mathrm{e}}$, and we can endow the graded $k$-vector space

$$
\mathbb{A}\left(B, \tau_{\Lambda^{\mathrm{e}}}\right)=\operatorname{Hom}_{\Lambda^{\mathrm{e}}}(B, B) \oplus \bigoplus_{i=1}^{\infty} \underline{\operatorname{Hom}}_{\Lambda^{\mathrm{e}}}\left(\tau_{\Lambda^{\mathrm{e}}}^{i}(B), B\right)
$$

with a product as follows; for two homogeneous elements $f \in \mathbb{A}\left(B, \tau_{\Lambda^{\mathrm{e}}}\right)_{m}$ and $g \in \mathbb{A}\left(B, \tau_{\Lambda^{\mathrm{e}}}\right)_{n}$ we define $f g$ to be the composition $f \circ \tau_{\Lambda^{\mathrm{e}}}^{m}(g) \in \mathbb{A}\left(B, \tau_{\Lambda^{\mathrm{e}}}\right)_{m+n}$. In this way $\mathbb{A}\left(B, \tau_{\Lambda^{\mathrm{e}}}\right)$ becomes a graded $k$-algebra. Now for any selfinjective Artin algebra $\Gamma$ the functors $\tau_{\Gamma}^{i}$ and $\Omega_{\Gamma}^{2 i} \mathcal{N}^{i}$ are isomorphic by ARS, Proposition IV.3.7], where $\mathcal{N}$ is the Nakayama functor $D \operatorname{Hom}_{\Gamma}(-, \Gamma)$. Therefore the orbit algebra $\mathbb{A}\left(B, \tau_{\Lambda^{\mathrm{e}}}\right)$ of the bimodule $B$ has the form

$$
\operatorname{Hom}_{\Lambda^{\mathrm{e}}}(B, B) \oplus \bigoplus_{i=1}^{\infty} \underline{\operatorname{Hom}}_{\Lambda^{\mathrm{e}}}\left(\Omega_{\Lambda^{\mathrm{e}}}^{2 i}\left(\mathcal{N}^{i} B\right), B\right),
$$

giving an isomorphism

$$
\mathbb{A}\left(B, \tau_{\Lambda^{\mathrm{e}}}\right) \simeq \bigoplus_{i=0}^{\infty} \operatorname{Ext}_{\Lambda^{\mathrm{e}}}^{2 i}\left(\mathcal{N}^{i} B, B\right)
$$

of graded $k$-algebras.

The algebra $\mathbb{A}\left(\Lambda, \tau_{\Lambda^{\mathrm{e}}}\right)$ is called the Auslander-Reiten orbit algebra of $\Lambda$, and these algebras have been extensively studied by Z. Pogorzały. In Po1] it was shown that they are invariant under stable equivalences of Morita type between symmetric algebras; if $\Gamma$ and $\Delta$ are finite dimensional symmetric $K$-algebras (where $K$ is a field) stably equivalent of Morita type, then $\mathbb{A}\left(\Gamma, \tau_{\Gamma^{\mathrm{e}}}\right)$ and $\mathbb{A}\left(\Delta, \tau_{\Delta^{\mathrm{e}}}\right)$ are isomorphic $K$-algebras. This was generalized in $[\mathrm{Po} 2$ to arbitrary finite dimensional selfinjective algebras. In $\mathrm{Po} 4$ the Auslander-Reiten orbit algebras of a class of finite dimensional basic connected selfinjective Nakayama $K$-algebras were computed. Namely, it was shown that if $\Gamma$ is such an algebra of $\tau_{\Gamma^{e}}$-period 1 , then $\mathbb{A}\left(\Gamma, \tau_{\Gamma^{\mathrm{e}}}\right) \simeq$ $K[x]$ if $\Gamma$ is a radical square zero algebra, and if not then there exists a natural number $t$ such that $\mathbb{A}\left(\Gamma, \tau_{\Gamma^{\mathrm{e}}}\right) \simeq K[x, y] /\left(y^{t}\right)$. In $[\mathrm{Po} 3]$-periodicity was investigated using similar techniques as was used in $\mathrm{Sch}$ to study syzygy-periodicity.

\section{Twisted Hochschild COHOMOLOGY}

The underlying geometric object used in EHSST and SnS to define support varieties was the Hochschild cohomology $\operatorname{ring} \operatorname{HH}^{*}(\Lambda, \Lambda)=\operatorname{Ext}_{\Lambda^{\mathrm{e}}}^{*}(\Lambda, \Lambda)=$ $\bigoplus_{n=0}^{\infty} \operatorname{Ext}_{\Lambda \mathrm{e}}^{n}(\Lambda, \Lambda)$ of $\Lambda$ (where $\Lambda^{\mathrm{e}}$ is the enveloping algebra $\Lambda \otimes_{k} \Lambda^{\text {op }}$ of $\Lambda$ ). For an Artin $k$-algebra $\Gamma$ and any pair of $\Lambda$ - $\Gamma$-bimodules $X$ and $Y$ the tensor map

$$
-\otimes_{\Lambda} X: \operatorname{Ext}_{\Lambda^{\mathrm{e}}}^{*}(\Lambda, \Lambda) \rightarrow \operatorname{Ext}_{\Lambda \otimes_{k} \Gamma^{\mathrm{op}}}(X, X)
$$

is a homomorphism of graded $k$-algebras, making $\operatorname{Ext}_{\Lambda \otimes_{k} \Gamma^{\circ p}}^{*}(X, Y)$ a left and right $\operatorname{Ext}_{\Lambda^{\mathrm{e}}}(\Lambda, \Lambda)$-module via the maps $-\otimes_{\Lambda} Y$ and $-\otimes_{\Lambda} X$, respectively (followed by Yoneda product). Now since $\Lambda$ is projective as a $k$-module, it follows from Yon, Proposition 3] that for any two $\Lambda$ - $\Lambda$ bimodules $B$ and $B^{\prime}$ which are both projective as right $\Lambda$-modules, and for any homogeneous elements $\eta \in \operatorname{Ext}_{\Lambda^{\mathrm{e}}}^{*}\left(B, B^{\prime}\right)$ and $\theta \in$ $\operatorname{Ext}_{\Lambda \otimes_{k} \Gamma^{\mathrm{op}}}(X, Y)$, the Yoneda relation

$$
\left(\eta \otimes_{\Lambda} Y\right) \circ\left(B \otimes_{\Lambda} \theta\right)=(-1)^{|\eta||\theta|}\left(B^{\prime} \otimes_{\Lambda} \theta\right) \circ\left(\eta \otimes_{\Lambda} X\right)
$$


holds (see also $[\mathrm{SnS}$, Theorem 1.1]). By specializing to the case $\Gamma=\Lambda$ and $B=$ $B^{\prime}=X=Y=\Lambda$ we see that the Hochshild cohomology ring $\operatorname{HH}^{*}(\Lambda, \Lambda)$ of $\Lambda$ is graded commutative, whereas the case $\Gamma=k$ and $B=B^{\prime}=\Lambda$ shows that for any pair of $\Lambda$-modules $X$ and $Y$ the left and right scalar actions from $\operatorname{HH}^{*}(\Lambda, \Lambda)$ on $\operatorname{Ext}_{\Lambda}^{*}(X, Y)$ are related graded commutatively.

Denote the commutative even subalgebra $\bigoplus_{n=0}^{\infty} \operatorname{Ext}_{\Lambda^{\mathrm{e}}}^{2 n}(\Lambda, \Lambda)$ of $\operatorname{HH}^{*}(\Lambda, \Lambda)$ by $H^{\mathrm{ev}}$. The support variety of the $\Lambda$-module $M$ is the subset

$$
\mathrm{V}(M) \stackrel{\text { def }}{=}\left\{\mathfrak{m} \in \operatorname{MaxSpec} H^{\mathrm{ev}} \mid \operatorname{Ann}_{H^{\mathrm{ev}}} \operatorname{Ext}_{\Lambda}^{*}(M, M) \subseteq \mathfrak{m}\right\}
$$

of the maximal ideal spectrum of $H^{\mathrm{ev}}$. As shown in EHSST] and [SnS], the theory of support varieties is rich and in many ways similar to the theory of cohomological varieties for groups, especially under the hypothesis that $H^{\mathrm{ev}}$ is Noetherian and that $\operatorname{Ext}_{\Lambda}^{*}(X, Y)$ is a finitely generated $H^{\mathrm{ev}}$-module for all $\Lambda$-modules $X$ and $Y$.

In order to obtain a partly similar theory of twisted support varieties, the underlying geometric object we use is a "twisted" version of the Hochschild cohomology ring. Let $\Gamma$ be any ring and let $\rho, \phi: \Gamma \rightarrow \Gamma$ be two ring automorphisms. If $X$ is a left $\Gamma$-module and $B$ is a $\Gamma$ - $\Gamma$-bimodule, denote by ${ }_{\phi} X$ and ${ }_{\phi} B_{\rho}$ the left module and bimodule whose scalar actions are defined by $\gamma \cdot x=\phi(\gamma) x$ and $\gamma_{1} \cdot b \cdot \gamma_{2}=\phi\left(\gamma_{1}\right) b \rho\left(\gamma_{2}\right)$ for $\gamma, \gamma_{1}, \gamma_{2} \in \Gamma, x \in X, b \in B$. The functor assigning to each $\Gamma$-module $X$ the twisted module ${ }_{\rho} X$ is exact and isomorphic to the functor ${ }_{\rho} \Gamma_{1} \otimes_{\Gamma}-$, and it preserves projective modules and minimal resolutions when the latter makes sense.

Fix a $k$-algebra automorphism

$$
\psi: \Lambda \rightarrow \Lambda
$$

and a positive integer $t \in \mathbb{N}$, and consider the graded $k$-module

$$
\operatorname{HH}^{t *}\left(\psi^{*} \Lambda_{1}, \Lambda\right)=\operatorname{Ext}_{\Lambda^{\mathrm{e}}}^{t *}\left(\psi^{*} \Lambda_{1}, \Lambda\right)=\bigoplus_{n=0}^{\infty} \operatorname{Ext}_{\Lambda^{\mathrm{e}}}^{t n}\left(\psi_{\psi^{n}} \Lambda_{1}, \Lambda\right) .
$$

By the above and Lemma 2.1 the pairing

$$
\begin{aligned}
\left.\operatorname{Ext}_{\Lambda^{\mathrm{e}}}^{t m}\left(\psi_{\psi^{m}} \Lambda_{1}, \Lambda\right) \times \operatorname{Ext}_{\Lambda^{\mathrm{e}}\left(\psi^{n}\right.}^{t n} \Lambda_{1}, \Lambda\right) & \rightarrow \operatorname{Ext}_{\Lambda^{\mathrm{e}}}^{t(m+n)}\left(\psi_{\psi^{m+n}} \Lambda_{1}, \Lambda\right) \\
(\eta, \theta) & \mapsto \eta{\psi^{m}}^{m} \theta
\end{aligned}
$$

defines a multiplication under which $\operatorname{HH}^{t *}\left(\psi^{*} \Lambda_{1}, \Lambda\right)$ becomes a graded $k$-algebra, i.e. if $\eta$ and $\theta$ are homogeneous elements given as exact sequences

$$
\begin{gathered}
\eta: 0 \rightarrow \Lambda \rightarrow E_{t m-1} \rightarrow \cdots \rightarrow E_{0} \rightarrow \psi^{m} \Lambda_{1} \rightarrow 0 \\
\theta: 0 \rightarrow \Lambda \rightarrow T_{t n-1} \rightarrow \cdots \rightarrow T_{0} \rightarrow \psi^{n} \Lambda_{1} \rightarrow 0,
\end{gathered}
$$

then their product $\eta \theta$ is given as the Yoneda product of the sequence $\eta$ with the sequence

$$
\psi^{m} \theta: 0 \rightarrow \psi^{m} \Lambda_{1} \rightarrow \psi^{m}\left(T_{t n-1}\right)_{1} \rightarrow \cdots \rightarrow \psi^{m}\left(T_{0}\right)_{1} \rightarrow \psi_{\psi^{m+n}} \Lambda_{1} \rightarrow 0 .
$$

Before giving an example, recall that a finite dimensional algebra $\Gamma$ over a field $K$ is Frobenius if ${ }_{\Gamma} \Gamma$ and $D\left(\Gamma_{\Gamma}\right)$ are isomorphic as left $\Gamma$-modules, where $D$ denotes the usual $K$-dual $\operatorname{Hom}_{K}(-, K)$. For such an algebra $\Gamma$, let $\varphi_{l}:{ }_{\Gamma} \Gamma \rightarrow D\left(\Gamma_{\Gamma}\right)$ be an isomorphism. Let $y \in \Gamma$ be any element, and consider the linear functional $\varphi_{l}(1) \cdot y \in D(\Gamma)$, i.e. the $K$-linear map $\Gamma \rightarrow K$ defined by $\gamma \mapsto \varphi_{l}(1)(y \gamma)$. Since $\varphi_{l}$ is surjective there is an element $x \in \Gamma$ having the property that $\varphi_{l}(x)=\varphi_{l}(1) \cdot y$, giving $x \cdot \varphi_{l}(1)=\varphi_{l}(1) \cdot y$ since $\varphi_{l}$ is a map of left $\Gamma$-modules. It is not difficult to show that the map $y \mapsto x$ defines a $K$-algebra automorphism on $\Gamma$, and its inverse $\nu_{\Gamma}$ is called the Nakayama automorphism of $\Gamma$ (with respect to $\varphi_{l}$ ). Thus $\nu_{\Gamma}$ is defined by $\varphi_{l}(1)(\gamma x)=\varphi_{l}(1)\left(\nu_{\Gamma}(x) \gamma\right)$ for all $\gamma \in \Gamma$. This automorphism is unique up to an inner automorphism; if $\varphi_{l}^{\prime}:{ }_{\Gamma} \Gamma \rightarrow D\left(\Gamma_{\Gamma}\right)$ is another isomorphism of left modules yielding a Nakayama automorphism $\nu_{\Gamma}^{\prime}$, then there exists an invertible 
element $z \in \Gamma$ such that $\nu_{\Gamma}=z \nu_{\Gamma}^{\prime} z^{-1}$. Note that $\varphi_{l}$ is an isomorphism between the bimodules ${ }_{1} \Gamma_{\nu_{\Gamma}^{-1}}$ and $D(\Gamma)$, and since $\nu_{\Gamma}^{-1}:{ }_{\nu_{\Gamma}} \Gamma_{1} \rightarrow_{1} \Gamma_{\nu_{\Gamma}^{-1}}$ is an isomorphism of bimodules we see that $\nu_{\Gamma} \Gamma_{1} \simeq D(\Gamma)$.

As $D\left(\Gamma_{\Gamma}\right)$ is an injective left $\Gamma$-module, we see that a Frobenuis algebra is always left selfinjective, but in fact the definition is left-right symmetric. For if $\varphi_{l}:{ }_{\Gamma} \Gamma \rightarrow D\left(\Gamma_{\Gamma}\right)$ is an isomorphism of left $\Gamma$-modules, we can dualize and get an isomorphism $D\left(\varphi_{l}\right): D^{2}\left(\Gamma_{\Gamma}\right) \rightarrow D\left({ }_{\Gamma} \Gamma\right)$ of right modules, and composing with the natural isomorphism $\Gamma_{\Gamma} \simeq D^{2}\left(\Gamma_{\Gamma}\right)$ we get an isomorphism $\varphi_{r}: \Gamma_{\Gamma} \rightarrow$ $D\left({ }_{\Gamma} \Gamma\right)$ of right $\Gamma$-modules. Moreover, we can view this last map as an isomorphism $\varphi_{r}: \Gamma^{\mathrm{op}} \Gamma^{\mathrm{op}} \rightarrow D\left(\Gamma_{\Gamma \mathrm{op}}^{\mathrm{op}}\right)$ of left $\Gamma^{\mathrm{op}}$-modules, thereby giving an isomorphism $\varphi_{l} \otimes \varphi_{r}: \Gamma \otimes_{K} \Gamma^{\mathrm{op}} \rightarrow D\left(\Gamma_{\Gamma}\right) \otimes_{K} D\left(\Gamma_{\Gamma_{\mathrm{op}}}^{\mathrm{op}}\right)$ of $\Gamma$ - $\Gamma$-bimodules. As $D\left(\Gamma_{\Gamma}\right) \otimes_{K} D\left(\Gamma_{\Gamma}^{\mathrm{op}}\right)$ is isomorphic to $D\left(\Gamma \otimes_{K} \Gamma^{\mathrm{op}}\right)$ as a $\Gamma$ - $\Gamma$-bimodule, we see that the enveloping algebra $\Gamma^{\mathrm{e}}=\Gamma \otimes_{K} \Gamma^{\mathrm{op}}$ of $\Gamma$ is also Frobenius.

Example. Suppose $k$ is a field and $\Lambda$ is Frobenius. Recall from the example in Section 2 that for a bimodule $B$ in the stable category $\underline{\bmod } \Lambda^{\mathrm{e}}$ the orbit algebra

$$
\mathbb{A}\left(B, \tau_{\Lambda^{\mathrm{e}}}\right)=\operatorname{Hom}_{\Lambda^{\mathrm{e}}}(B, B) \oplus \bigoplus_{i=1}^{\infty} \underline{\operatorname{Hom}}_{\Lambda^{\mathrm{e}}}\left(\tau_{\Lambda^{\mathrm{e}}}^{i}(B), B\right)
$$

is isomorphic to the algebra

$$
\bigoplus_{i=0}^{\infty} \operatorname{Ext}_{\Lambda^{\mathrm{e}}}^{2 i}\left(\mathcal{N}^{i} B, B\right)
$$

where $\mathcal{N}$ is the Nakayama functor $D \operatorname{Hom}_{\Lambda^{\mathrm{e}}}\left(-, \Lambda^{\mathrm{e}}\right)$. This functor maps the projective cover of a simple $\Lambda^{\mathrm{e}}$-module to its injective envelope, and is therefore isomorphic as a functor to $D\left(\Lambda^{\mathrm{e}}\right) \otimes_{\Lambda^{\mathrm{e}}}-$. As $\Lambda$ is a Frobenius algebra, so is $\Lambda^{\mathrm{e}}$, and we know that $D\left(\Lambda^{\mathrm{e}}\right)$ is isomorphic to $\nu_{\Lambda^{\mathrm{e}}} \Lambda^{\mathrm{e}}{ }_{1}$ as a $\Lambda^{\mathrm{e}}-\Lambda^{\mathrm{e}}$-bimodule, giving an isomorphism

of graded $k$-algebras.

$$
\mathbb{A}\left(B, \tau_{\Lambda^{\mathrm{e}}}\right) \simeq \bigoplus_{i=0}^{\infty} \operatorname{Ext}_{\Lambda^{\mathrm{e}}}^{2 i}\left(\nu_{\Lambda^{\mathrm{e}}}^{i} B, B\right)
$$

Next we address the question of commutativity in $\mathrm{HH}^{t *}\left(\psi_{*} \Lambda_{1}, \Lambda\right)$. For two homogeneous elements

$$
\begin{array}{r}
\eta: 0 \rightarrow \Lambda \rightarrow E_{t m-1} \rightarrow \cdots \rightarrow E_{0} \rightarrow \psi^{m} \Lambda_{1} \rightarrow 0 \\
\theta: 0 \rightarrow \Lambda \rightarrow T_{t n-1} \rightarrow \cdots \rightarrow T_{0} \rightarrow \psi^{n} \Lambda_{1} \rightarrow 0
\end{array}
$$

the Yoneda relation (†) gives the equality

$$
\eta \circ\left({ }_{\psi^{m}} \Lambda_{1} \otimes_{\Lambda} \theta\right)=(-1)^{|\eta||\theta|} \theta \circ\left(\eta \otimes_{\Lambda} \psi^{n} \Lambda_{1}\right),
$$

in which the left hand side is the product $\eta \theta$ (we can identify $\psi^{m} \Lambda_{1} \otimes_{\Lambda} \theta$ with $\left.\psi^{m} \theta\right)$. However, the extension on the right hand side is in general not the product $\theta \eta$; it is the Yoneda product of $\theta$ with $\eta_{\psi^{-n}}$, and although we have bimodule isomorphisms ${ }_{1} \Lambda_{\psi^{-n}} \simeq \psi_{\psi^{n}} \Lambda_{1}$ and $\psi_{\psi^{m}} \Lambda_{\psi^{-n}} \simeq \psi_{\psi^{m+n}} \Lambda_{1}$ we cannot in general identify $\eta_{\psi^{-n}}$ with $\psi^{n} \eta$ unless $n=0$. In fact, the following example shows that the algebra $\mathrm{HH}^{t *}\left(\psi_{*}^{*} \Lambda_{1}, \Lambda\right)$ in general is not graded commutative.

Example. Suppose $k$ is an algebraically closed field not of characteristic 2, and for any natural numbers $m$ and $n$, with $n \geq 2$, let $Q_{m, n}$ be the quiver

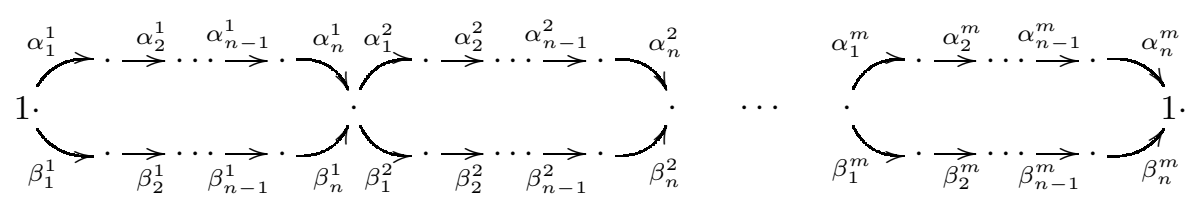


in which the leftmost vertex is the same as the rightmost vertex. Denote by $\mathfrak{a}_{m, n}$ the ideal in the path algebra $k Q_{m, n}$ generated by the paths of length $n+1$, the sums of the parallel paths of length $n$, and the paths $\left\{\beta_{1}^{1} \alpha_{n}^{m}, \alpha_{1}^{1} \beta_{n}^{m}, \beta_{1}^{i+1} \alpha_{n}^{i}, \alpha_{1}^{i+1} \beta_{n}^{i}\right\}_{i=1}^{m-1}$. Finally, denote the finite dimensional algebra $k Q_{m, n} / \mathfrak{a}_{m, n}$ by $\Lambda_{m, n}$. It was shown in Rie] and $\left[\mathrm{ScW}\right.$ ] that the algebras $\left\{\Lambda_{m, n}\right\}_{1 \leq m, 2 \leq n}$ represent the distinct stable equivalence classes of Möbius algebras. Moreover, in [Asa and [M-H] it was shown that these algebras also represent the distinct derived equivalent classes of Möbius algebras.

Let $\Lambda$ be an algebra of the above type. By [EHS, Theorem 3.5] there is an automorphism $\Lambda \stackrel{\psi}{\rightarrow} \Lambda$ such that $\Omega_{\Lambda^{\mathrm{e}}}^{p}(\Lambda) \simeq \psi_{\psi^{-1}} \Lambda_{1}$, where $p=2 n-1$. Thus the initial part of the minimal bimodule resolution of $\Lambda$ has the form

$$
0 \rightarrow \psi^{-1} \Lambda_{1} \rightarrow Q_{p-1} \rightarrow \cdots \rightarrow Q_{0} \rightarrow \Lambda \rightarrow 0
$$

and twisting this exact sequence with $\psi$ we obtain the initial part

$$
0 \rightarrow \Lambda \rightarrow{ }_{\psi}\left(Q_{p-1}\right)_{1} \rightarrow \cdots \rightarrow_{\psi}\left(Q_{0}\right)_{1} \rightarrow_{\psi} \Lambda_{1} \rightarrow 0
$$

of the minimal bimodule resolution of ${ }_{\psi} \Lambda_{1}$. The latter exact sequence corresponds to an element $\mu$ in $\mathrm{HH}^{p *}\left(\psi_{\psi^{*}} \Lambda_{1}, \Lambda\right)$, and if this algebra is graded commutative then the relation $\mu^{2}=(-1)^{p^{2}} \mu^{2}$ implies $\mu^{2}=0$ (since $p$ is odd). However, since $\Omega_{\Lambda^{\mathrm{e}}}^{2 p}\left(\psi_{\psi^{2}} \Lambda_{1}\right)=\Lambda$, we may represent the element $\mu^{2} \in \operatorname{Ext}_{\Lambda^{\mathrm{e}}}^{2 p}\left(\psi_{\psi^{2}} \Lambda_{1}, \Lambda\right)$ by the identity map $\Omega_{\Lambda^{\mathrm{e}}}^{2 p}\left(\psi_{\psi^{2}} \Lambda_{1}\right) \stackrel{\text { id }}{\rightarrow} \Lambda$, and so if $\mu^{2}=0$ then the identity on $\Lambda$ factors through a projective bimodule. Then $\Lambda$ itself is projective as a bimodule, and therefore semisimple as an algebra. This is obviously impossible, hence the algebra $\mathrm{HH}^{p *}\left(\psi^{*} \Lambda_{1}, \Lambda\right)$ cannot be graded commutative.

The above example shows that the twisted Hochschild cohomology ring in general is not graded commutative. In light of this example and the Yoneda relation (†), all we can say about commutativity in $\operatorname{HH}^{t *}\left(\psi_{*} \Lambda_{1}, \Lambda\right)$ is that a homogeneous degree zero element commutes with everything, which is not unexpected since $\operatorname{HH}^{0}\left(\psi_{\psi^{0}} \Lambda_{1}, \Lambda\right)=\operatorname{Ext}_{\Lambda^{\mathrm{e}}}^{0}(\Lambda, \Lambda)=\mathrm{Z}(\Lambda)$ (the center of $\Lambda$ ). The next result gives a criterion under which commutativity relations hold, but first recall the following from [CaE, page 174-175]: for each $n \geq 0$ let $Q_{n}$ denote the $n$-fold tensor product of $\Lambda$ over $k$ (with $Q_{0}=k$ ), and define

$$
B^{n}=Q_{n+2}=\underbrace{\Lambda \otimes_{k} \cdots \otimes_{k} \Lambda}_{n+2 \text { copies of } \Lambda} .
$$

We give $B^{n}$ a $\Lambda^{\mathrm{e}}$-module structure by defining

$$
\lambda\left(\lambda_{0} \otimes \cdots \otimes \lambda_{n+1}\right) \lambda^{\prime}=\lambda \lambda_{0} \otimes \cdots \otimes \lambda_{n+1} \lambda^{\prime},
$$

and as $A^{\mathrm{e}}$-modules we then have an isomorphism $B^{n} \simeq \Lambda^{\mathrm{e}} \otimes_{k} Q_{n}$. Note that $B^{n}$ is $\Lambda^{\mathrm{e}}$-projective since the functor $\operatorname{Hom}_{\Lambda^{\mathrm{e}}}\left(\Lambda^{\mathrm{e}} \otimes_{k} Q_{n},-\right)$ is naturally isomorphic to $\operatorname{Hom}_{k}\left(Q_{n},-\right)$ by adjointness. Now for each $n \geq 1$, define $d: B^{n} \rightarrow B^{n-1}$ by

$$
\lambda_{0} \otimes \cdots \otimes \lambda_{n+1} \mapsto \sum_{i=0}^{n}(-1)^{i} \lambda_{0} \otimes \cdots \otimes \lambda_{i} \lambda_{i+1} \otimes \cdots \otimes \lambda_{n+1}
$$

The sequence

$$
\mathbb{B}: \cdots \rightarrow B^{3} \stackrel{d}{\rightarrow} B^{2} \stackrel{d}{\rightarrow} B^{1} \stackrel{d}{\rightarrow} B^{0} \stackrel{\mu}{\rightarrow} \Lambda \rightarrow 0,
$$

where $\mu$ is the "multiplication map", is an exact $\Lambda^{\mathrm{e}}$-projective resolution called the standard resolution (or Bar-resolution) of $\Lambda$.

Proposition 3.1. Let $\eta \in \mathrm{HH}^{t *}\left(\psi_{\psi^{*}} \Lambda_{1}, \Lambda\right)$ be a homogeneous element of degree $\mathrm{tm}$ represented by the map $f_{\eta}: \psi_{\psi^{m}} B_{1}^{t m} \rightarrow \Lambda$. If for some $n \geq 1$

$$
f_{\eta}\left(\psi^{-n}\left(\lambda_{0}\right) \otimes \cdots \otimes \psi^{-n}\left(\lambda_{t m}\right) \otimes 1\right)=\psi^{-n} f_{\eta}\left(\lambda_{0} \otimes \cdots \otimes \lambda_{t m} \otimes 1\right)
$$


for all $\lambda_{0} \otimes \cdots \otimes \lambda_{t m} \otimes 1$ in $\psi_{\psi^{m}} B_{1}^{t m}$, then $\eta_{\psi^{-n}}=\psi_{\psi^{n}} \eta$, and consequently $\eta \theta=$ $(-1)^{|\eta||\theta|} \theta \eta$ for every homogeneous element $\theta \in \mathrm{HH}^{t *}\left(\psi_{\psi^{*}} \Lambda_{1}, \Lambda\right)$ such that $t n$ divides $|\theta|$. In particular, if

$$
f_{\eta}\left(\psi^{-1}\left(\lambda_{0}\right) \otimes \cdots \otimes \psi^{-1}\left(\lambda_{t m}\right) \otimes 1\right)=\psi^{-1} f_{\eta}\left(\lambda_{0} \otimes \cdots \otimes \lambda_{t m} \otimes 1\right)
$$

for all $\lambda_{0} \otimes \cdots \otimes \lambda_{t m} \otimes 1$ in $\psi^{m} B_{1}^{t m}$, then $\eta$ belongs to the graded center of $\mathrm{HH}^{t *}\left(\psi_{*} \Lambda_{1}, \Lambda\right)$.

Proof. Suppose the given condition on $f_{\eta}$ holds for $n$. When viewing $\eta_{\psi^{-n}}$ as a $t m$-fold extension of $\psi^{n} \Lambda_{1}$ by $\psi^{m+n} \Lambda_{1}$, we use the bimodule isomorphisms $\psi^{-n}: \psi^{m+n} \Lambda_{1} \rightarrow \psi_{\psi^{m}} \Lambda_{\psi^{-n}}$ and $\psi^{n}:{ }_{1} \Lambda_{\psi^{-n}} \rightarrow \psi_{\psi^{n}} \Lambda_{1}$. A lifting of the former along $\psi^{m+n} \mathbb{B}_{1}$ is given by the maps

$$
\begin{aligned}
\left(\psi^{-n}\right)^{\otimes i+2}:{ }_{\psi^{m+n}} B_{1}^{i} & \rightarrow \psi^{m} B_{\psi^{-n}}^{i} \\
\lambda_{0} \otimes \cdots \otimes \lambda_{i+1} & \mapsto \psi^{-n}\left(\lambda_{0}\right) \otimes \cdots \otimes \psi^{-n}\left(\lambda_{i+1}\right)
\end{aligned}
$$

for $i \geq 0$, giving the commutative diagram

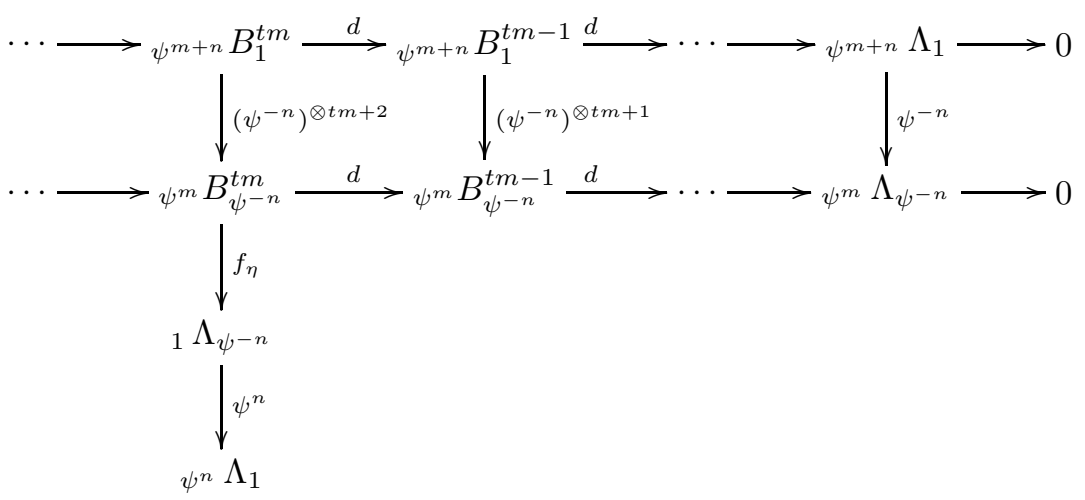

with exact rows. Therefore the extension $\eta_{\psi^{-n}}$ is represented by the map composite map

$$
\psi^{n} \circ f_{\eta} \circ\left(\psi^{-n}\right)^{\otimes t m+2}: \psi^{m+n} B_{1}^{t m} \rightarrow_{\psi^{n}} \Lambda_{1},
$$

under which the image of an element $\lambda_{0} \otimes \cdots \otimes \lambda_{t m+1} \in_{\psi^{m+n}} B_{1}^{t m}$ is easily seen to be $f_{\eta}\left(\lambda_{0} \otimes \cdots \otimes \lambda_{t m+1}\right)$ because of the assumption on $f_{\eta}$.

This shows that we may identify the extensions $\eta_{\psi^{-n}}$ and $\psi_{\psi^{n}} \eta$, and by induction we see that $\eta_{\psi^{-j n}}={ }_{\psi^{j n}} \eta$ for all $j \geq 1$. Consequently, if $\theta \in \operatorname{HH}^{t *}\left(\psi^{*} \Lambda_{1}, \Lambda\right)$ is a homogeneous element such that $t n$ divides $|\theta|$, say $|\theta|=t n j$, then the Yoneda relation gives

$$
\eta \theta=(-1)^{|\eta||\theta|} \theta \circ \eta_{\psi^{-j n}}=(-1)^{|\eta||\theta|} \theta \circ \psi_{\psi^{j n}} \eta=(-1)^{|\eta||\theta|} \theta \eta .
$$

For reasons to be explained in the next section, we make the following definition motivated by the above proposition.

Definition 3.2. A commutative graded subalgebra $H \subseteq \operatorname{HH}^{t *}\left(\psi_{\psi^{*}} \Lambda_{1}, \Lambda\right)$ is strongly commutative if $\eta_{\psi^{-1}}={ }_{\psi} \eta$ for all homogeneous elements $\eta \in H$.

We end this section with an example of a Frobenius algebra for which the twisted Hochschild cohomology ring with respect to the Nakayama automorphism and $t=2$ is strongly commutative.

Example. Let $k$ be a field not of characteristic 2 and $q \in k$ a nonzero element which is not a root of unity, and denote by $\Lambda$ the $k$-algebra

$$
\Lambda=k\langle x, y\rangle /\left(x^{2}, x y+q y x, y^{2}\right) .
$$


Denote by $D$ the usual $k$-dual $\operatorname{Hom}_{k}(-, k)$, and consider the map $\varphi:{ }_{\Lambda} \Lambda \rightarrow D\left(\Lambda_{\Lambda}\right)$ of left $\Lambda$-modules defined by

$$
\varphi(1)(\alpha+\beta x+\gamma y+\delta y x) \stackrel{\text { def }}{=} \delta .
$$

It is easy to show that this is an injective map and hence also an isomorphism since $\operatorname{dim}_{k} \Lambda=\operatorname{dim}_{k} D(\Lambda)$, and therefore $\Lambda$ is a Frobenius algebra by definition. Straightforward calculations show that $x \cdot \varphi(1)=\varphi(1) \cdot\left(-q^{-1} x\right)$ and $y \cdot \varphi(1)=$ $\varphi(1) \cdot(-q y)$, hence since $x$ and $y$ generate $\Lambda$ over $k$ we see that the Nakayama automorphism $\nu$ (with respect to $\varphi$ ) is the degree preserving map defined by

$$
x \mapsto-q^{-1} x, \quad y \mapsto-q y .
$$

In $\mathrm{Be} 2$ the Hochschild cohomology of this 4-dimensional graded Koszul algebra was studied, and for every degree preserving $k$-algebra automorphism $\psi: \Lambda \rightarrow \Lambda$ the cohomology groups $\operatorname{HH}^{*}\left(\Lambda,{ }_{1} \Lambda_{\psi}\right)=\operatorname{Ext}_{\Lambda^{\mathrm{e}}}\left(\Lambda,{ }_{1} \Lambda_{\psi}\right)$ were computed. In particular, from $[\mathrm{Be} 2,3.4(\mathrm{iv})]$ we get

$$
\operatorname{dim}_{k} \operatorname{Ext}_{\Lambda^{\mathrm{e}}}^{2 n}\left(\Lambda,{ }_{1} \Lambda_{\nu^{n}}\right)= \begin{cases}1 & \text { for } n \text { even } \\ 0 & \text { for } n \text { odd }\end{cases}
$$

when $n>0$, whereas $\operatorname{Ext}_{\Lambda^{\mathrm{e}}}^{0}\left(\Lambda,{ }_{1} \Lambda_{\nu^{0}}\right)=\mathrm{Z}(\Lambda)$ is two dimensional (the elements 1 and $y x$ form a basis for the center of $\Lambda$ ). Moreover, it is not difficult to see that $\operatorname{Ext}_{\Lambda^{\mathrm{e}}}^{2 n}\left(\Lambda,{ }_{1} \Lambda_{\nu^{n}}\right)$ is isomorphic to $\operatorname{Ext}_{\Lambda^{\mathrm{e}}}^{2 n}\left({ }_{\nu^{n}} \Lambda_{1}, \Lambda\right)$. Therefore the algebra $\operatorname{HH}^{4 *}\left({ }_{\nu^{2 *}} \Lambda_{1}, \Lambda\right)=\bigoplus_{n=0}^{\infty} \operatorname{Ext}_{\Lambda^{\mathrm{e}}}^{4 n}\left({ }_{\nu^{2 n}} \Lambda_{1}, \Lambda\right)$ is two dimensional in degree zero and one dimensional in the positive degrees.

We now recall the construction of the minimal bimodule projective resolution of $\Lambda$ from [BGMS]. Define the elements

$$
\begin{gathered}
f_{0}^{0}=1, \quad f_{0}^{1}=x, \quad f_{1}^{1}=y, \\
f_{-1}^{n}=0=f_{n+1}^{n} \text { for each } n \geq 0,
\end{gathered}
$$

and for each $n \geq 2$ define elements $\left\{f_{i}^{n}\right\}_{i=0}^{n} \subseteq \underbrace{\Lambda \otimes_{k} \cdots \otimes_{k} \Lambda}_{n \text { copies }}$ inductively by

$$
f_{i}^{n}=f_{i-1}^{n-1} \otimes y+q^{i} f_{i}^{n-1} \otimes x .
$$

Denote by $F^{n}$ the $\Lambda^{\mathrm{e}}$-projective module $\bigoplus_{i=0}^{n} \Lambda \otimes_{k} f_{i}^{n} \otimes_{k} \Lambda$, and by $\tilde{f}_{i}^{n}$ the element $1 \otimes f_{i}^{n} \otimes 1 \in F^{n}$ (and $\tilde{f}_{0}^{0}=1 \otimes 1$ ). The set $\left\{\tilde{f}_{i}^{n}\right\}_{i=0}^{n}$ generates $F^{n}$ as a $\Lambda^{\mathrm{e}}$-module. Now define a map $\delta: F^{n} \rightarrow F^{n-1}$ by

$$
\tilde{f}_{i}^{n} \mapsto\left[x \tilde{f}_{i}^{n-1}+(-1)^{n} q^{i} \tilde{f}_{i}^{n-1} x\right]+\left[q^{n-i} y \tilde{f}_{i-1}^{n-1}+(-1)^{n} \tilde{f}_{i-1}^{n-1} y\right] .
$$

It is shown in BGMS that

$$
(\mathbb{F}, \delta): \cdots \rightarrow F^{n+1} \stackrel{\delta}{\rightarrow} F^{n} \stackrel{\delta}{\rightarrow} F^{n-1} \rightarrow \cdots
$$

is a minimal $\Lambda^{\mathrm{e}}$-projective resolution of $\Lambda$.

For each $m \geq 1$ consider the $\Lambda^{\mathrm{e}}$-linear map

$$
\begin{aligned}
g_{4 m}: \nu^{2 m} F_{1}^{4 m} & \rightarrow \Lambda \\
\tilde{f}_{i}^{4 m} & \mapsto \begin{cases}1 & \text { for } i=2 m \\
0 & \text { for } i \neq 2 m .\end{cases}
\end{aligned}
$$

The only generators in $\nu^{2 m} F_{1}^{4 m+1}$ which can possibly map to $\tilde{f}_{2 m}^{4 m}$ under the map $\nu^{2 m} F_{1}^{4 m+1} \stackrel{\delta}{\rightarrow} \nu^{2 m} F_{1}^{4 m}$ are $\tilde{f}_{2 m}^{4 m+1}$ and $\tilde{f}_{2 m+1}^{4 m+1}$, and so it follows from the equalities

$$
\begin{aligned}
g_{4 m} \circ \delta\left(\tilde{f}_{2 m}^{4 m+1}\right) & =g_{4 m}\left(x \tilde{f}_{2 m}^{4 m}-q^{2 m} \tilde{f}_{2 m}^{4 m} x\right) \\
& =g_{4 m}\left(\left[q^{2 m} x\right] \cdot \tilde{f}_{2 m}^{4 m}-\tilde{f}_{2 m}^{4 m} \cdot\left[q^{2 m} x\right]\right) \\
& =0
\end{aligned}
$$


and

$$
\begin{aligned}
g_{4 m} \circ \delta\left(\tilde{f}_{2 m+1}^{4 m+1}\right) & =g_{4 m}\left(q^{2 m} y \tilde{f}_{2 m}^{4 m}-\tilde{f}_{2 m}^{4 m} y\right) \\
& =g_{4 m}\left(y \cdot \tilde{f}_{2 m}^{4 m}-\tilde{f}_{2 m}^{4 m} \cdot y\right) \\
& =0
\end{aligned}
$$

that $g_{4 m}$ belongs to the kernel of the map $\delta^{*}: \operatorname{Hom}_{\Lambda^{\mathrm{e}}}\left({ }_{\nu^{2 m}} F_{1}^{4 m}, \Lambda\right) \rightarrow$ $\operatorname{Hom}_{\Lambda^{\mathrm{e}}}\left({ }_{\nu^{2 m}} F_{1}^{4 m+1}, \Lambda\right)$. Moreover, there cannot exist a $\Lambda^{\mathrm{e}}$-linear map $\nu^{2 m} F_{1}^{4 m-1} \rightarrow$ $\Lambda$ making the diagram

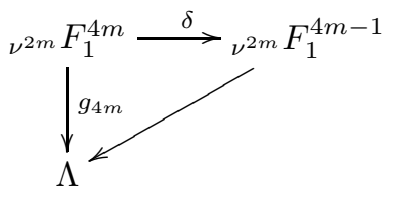

commute, since $\delta$ is a map of graded degree 1 , whereas the degree of $g_{4 m}$ is 0 . This shows that $g_{4 m}$ represents a generator for the one dimensional space $\operatorname{Ext}_{\Lambda^{\mathrm{e}}}^{4 m}\left({ }_{\nu^{2 m}} \Lambda_{1}, \Lambda\right)$. Note that if we multiply this map with $y x$, then there does exist a map making the above diagram commute, hence whenever we multiply any homogeneous element of positive degree in $\operatorname{HH}^{4 *}\left({ }_{\nu^{2 *}} \Lambda_{1}, \Lambda\right)$ with the element $y x \in \mathrm{HH}^{0}\left({ }_{\nu^{0}} \Lambda_{1}, \Lambda\right)$ we get zero.

For each $0 \leq i \leq 4$ define $\Lambda^{\mathrm{e}}$-linear maps $\bar{g}_{4 m+i}:{ }_{\nu^{2(m+1)}} F_{1}^{4 m+i} \rightarrow_{\nu^{2}} F_{1}^{i}$ by

$$
\begin{aligned}
\bar{g}_{4 m}: \tilde{f}_{i}^{4 m} & \mapsto \begin{cases}\tilde{f}_{0}^{0} \text { for } i=2 m \\
0 & \text { otherwise }\end{cases} \\
\bar{g}_{4 m+1}: \tilde{f}_{i}^{4 m+1} & \mapsto \begin{cases}q^{2 m} \tilde{f}_{0}^{1} & \text { for } i=2 m \\
\tilde{f}_{1}^{1} & \text { for } i=2 m+1 \\
0 & \text { otherwise }\end{cases} \\
\bar{g}_{4 m+2}: \tilde{f}_{i}^{4 m+2} & \mapsto \begin{cases}q^{4 m} \tilde{f}_{0}^{2} & \text { for } i=2 m \\
q^{2 m} \tilde{f}_{1}^{2} & \text { for } i=2 m+1 \\
\tilde{f}_{2}^{2} & \text { for } i=2 m+2 \\
0 & \text { otherwise }\end{cases} \\
\bar{g}_{4 m+3}: \tilde{f}_{i}^{4 m+3} & \mapsto \begin{cases}q^{4 m} \tilde{f}_{1}^{3} & \text { for } i=2 m+1 \\
q^{2 m} \tilde{f}_{2}^{3} & \text { for } i=2 m+2 \\
0 & \text { otherwise }\end{cases} \\
\bar{g}_{4 m+4}: \tilde{f}_{i}^{4 m+4} & \mapsto \begin{cases}q^{4 m} \tilde{f}_{2}^{4} & \text { for } i=2 m \\
0 & \text { otherwise. }\end{cases}
\end{aligned}
$$

Then for each $1 \leq i \leq 4$ the diagram

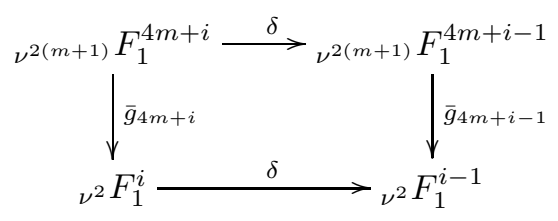

commutes, hence the maps $\bar{g}_{4 m+i}$ for $0 \leq i \leq 4$ provide a lifting of $\nu^{2(m+1)} F_{1}^{4 m} \stackrel{\nu^{2} g_{4 m}}{\longrightarrow} \nu^{2} \Lambda_{1}$ along the projective bimodule resolution $\nu^{2(m+1)} \mathbb{F}_{1}$ of $\nu^{2(m+1)} \Lambda_{1}$. Moreover, the composition $g_{4} \circ \bar{g}_{4 m+4}$ equals the map $q^{4 m} g_{4 m+4}$. Therefore, if we denote by $\theta$ the extension in $\operatorname{Ext}_{\Lambda^{\mathrm{e}}}^{4}\left({ }_{\nu^{2}} \Lambda_{1}, \Lambda\right)$ corresponding to the map $g_{4}$, we see that for each $m \geq 2$ the element $\theta^{m}$ is the (nonzero) extension in $\operatorname{Ext}_{\Lambda^{\mathrm{e}}}^{4 m}\left({ }_{\nu^{2 m}} \Lambda_{1}, \Lambda\right)$ corresponding to the map $q^{4(1+2+\cdots+m-1)} g_{4 m}$.

As a result, we see that we have an isomorphism

$$
\mathrm{HH}^{4 *}\left({ }_{\nu}{ }^{2 *} \Lambda_{1}, \Lambda\right) \simeq k[x] \times_{k} k
$$


of graded $k$-algebras, with $x$ of degree 4 , and in particular $\operatorname{HH}^{4 *}\left({ }_{\nu^{2 *}} \Lambda_{1}, \Lambda\right)$ is commutative. Moreover, it is easily shown that we may identify the extensions $\theta_{\nu^{-2}}$ and ${ }_{\nu^{2}} \theta$, and so $\mathrm{HH}^{4 *}\left(\nu_{\nu^{2 *}} \Lambda_{1}, \Lambda\right)$ is actually strongly commutative.

\section{TWISTED SUPPORT VARIETIES}

In order to obtain a theory of twisted support varieties, the underlying geometric object we shall use is the twisted Hochschild cohomology ring $\operatorname{HH}^{t *}\left(\psi_{*} \Lambda_{1}, \Lambda\right)=$ $\operatorname{Ext}_{\Lambda^{\mathrm{e}}}^{t *}\left(\psi^{*} \Lambda_{1}, \Lambda\right)$ studied in the previous section (where $\Lambda \stackrel{\psi}{\rightarrow} \Lambda$ is an automorphism and $t \in \mathbb{N})$. Similarly as for $\operatorname{HH}^{t *}\left(\psi^{*} \Lambda_{1}, \Lambda\right)$, the graded $k$-module $\operatorname{Ext}_{\Lambda}^{t *}\left(\psi^{*} M, M\right)=$ $\bigoplus_{n=0}^{\infty} \operatorname{Ext}_{\Lambda}^{t n}\left(\psi^{n} M, M\right)$ is a $k$-algebra, and for each $\Lambda$-module $N$ the graded $k$-module $\operatorname{Ext}_{\Lambda}^{t *}\left(\psi^{*} M, N\right)=\bigoplus_{n=0}^{\infty} \operatorname{Ext}_{\Lambda}^{t n}\left(\psi^{n} M, N\right)$ becomes a graded right $\operatorname{Ext}_{\Lambda}^{t *}\left(\psi^{*} M, M\right)$ module by defining

$$
\zeta \mu \stackrel{\text { def }}{=} \zeta \circ_{\psi^{m}} \mu
$$

for $\zeta \in \operatorname{Ext}_{\Lambda}^{t m}\left(\psi_{\psi^{m}} M, N\right)$ and $\mu \in \operatorname{Ext}_{\Lambda}^{t n}\left(\psi_{\psi^{n}} M, M\right)$. Moreover, the tensor map

$$
\begin{aligned}
-\otimes_{\Lambda} M: \operatorname{HH}^{t *}\left(\psi^{*} \Lambda_{1}, \Lambda\right) & \rightarrow \operatorname{Ext}_{\Lambda}^{t *}\left(\psi^{*} M, M\right) \\
\eta & \mapsto \eta \otimes_{\Lambda} M
\end{aligned}
$$

is a homomorphism of graded $k$-algebras, thus making $\operatorname{Ext}_{\Lambda}^{t *}\left(\psi^{*} M, N\right)$ a graded right $\mathrm{HH}^{t *}\left(\psi^{*} \Lambda_{1}, \Lambda\right)$-module. Similarly $\operatorname{Ext}_{\Lambda}^{t *}\left(\psi^{*} M, N\right)$ becomes a graded left $\mathrm{HH}^{t *}\left(\psi_{*} \Lambda_{1}, \Lambda\right)$ via the homomorphism

$$
\begin{aligned}
-\otimes_{\Lambda} N: \operatorname{HH}^{t *}\left(\psi^{*} \Lambda_{1}, \Lambda\right) & \rightarrow \operatorname{Ext}_{\Lambda}^{t *}\left(\psi^{*} N, N\right) \\
\eta & \mapsto \eta \otimes_{\Lambda} N
\end{aligned}
$$

of algebras.

We now make the following assumption:

Assumption. Given the automorphism $\Lambda \stackrel{\psi}{\rightarrow} \Lambda$ and the integer $t \in \mathbb{N}$, the graded subalgebra $H=\bigoplus_{n=0}^{\infty} H^{t n}$ of $\operatorname{HH}^{t *}\left(\psi^{*} \Lambda_{1}, \Lambda\right)$ is strongly commutative with $H^{0}=$ $\mathrm{HH}^{0}(\Lambda, \Lambda)=\mathrm{Z}(\Lambda)$.

Why do we restrict ourselves to strongly commutative algebras instead of "ordinary" commutative algebras? The answer is that we want the bifunction $\operatorname{Ext}_{\Lambda}^{t *}\left(\psi^{*}-,-\right)$, which maps the pair $(M, N)$ of $\Lambda$-modules to the $H$-module $\operatorname{Ext}_{\Lambda}^{t *}\left(\psi^{*} M, N\right)$, to preserve maps. Namely, consider two homogeneous elements $\zeta \in \operatorname{Ext}_{\Lambda}^{t m}\left(\psi^{m} M, N\right)$ and $\eta \in \operatorname{Ext}_{\Lambda^{\mathrm{e}}}^{t n}\left(\psi^{n} \Lambda_{1}, \Lambda\right)$. By definition $\zeta \cdot \eta$ is the extension $\zeta \circ \psi_{\psi^{m}}\left(\eta \otimes_{\Lambda} M\right)=\zeta \circ\left(\psi_{\psi^{m}} \eta \otimes_{\Lambda} M\right)$, and if $\psi_{\psi^{m}} \eta=\eta_{\psi^{-m}}$ we get $\psi^{m} \eta \otimes_{\Lambda} M=$ $\eta_{\psi^{-m}} \otimes_{\Lambda} M=\eta \otimes_{\Lambda} \psi^{m} M$. Hence in this case we may identify the right scalar product $\zeta \cdot \eta$ with $\left(\Lambda \otimes_{\Lambda} \zeta\right) \circ\left(\eta \otimes_{\Lambda} \psi_{m} M\right)$, and then by the Yoneda relation (†) from the previous section we get

$$
\zeta \cdot \eta=\left(\Lambda \otimes_{\Lambda} \zeta\right) \circ\left(\eta \otimes_{\Lambda} \psi^{m} M\right)=(-1)^{|\zeta||\eta|}\left(\eta \otimes_{\Lambda} N\right) \circ\left(\psi^{n} \Lambda_{1} \otimes_{\Lambda} \zeta\right) .
$$

However, the extension $\left(\eta \otimes_{\Lambda} N\right) \circ\left(\psi^{n} \Lambda_{1} \otimes_{\Lambda} \zeta\right)$ is precisely the left scalar product $\eta \cdot \zeta$, and so if $H$ is strongly commutative we see that

$$
\zeta \cdot \eta=(-1)^{|\zeta||\eta|} \eta \cdot \zeta
$$

for all homogeneous elements $\eta \in H$ and $\zeta \in \operatorname{Ext}_{\Lambda}^{t *}\left(\psi_{\psi^{*}} M, N\right)$.

The fact that the left and right scalar multiplications basically coincide is what makes $\operatorname{Ext}_{\Lambda}^{t *}\left(\psi^{*}-,-\right)$ preserve maps. To see this, let $f: M \rightarrow M^{\prime}$ be a $\Lambda$-homomorphism. This map induces a homomorphism $\hat{f}: \operatorname{Ext}_{\Lambda}^{t *}\left(\psi_{*}^{*} M^{\prime}, N\right) \rightarrow$ $\operatorname{Ext}_{\Lambda}^{t *}\left(\psi^{*} M, N\right)$ of graded groups, under which the image of a homogeneous element

$$
\theta: 0 \rightarrow N \rightarrow X_{t n} \rightarrow \cdots \rightarrow X_{1} \rightarrow \psi^{n} M^{\prime} \rightarrow 0
$$


is the extension $\theta f$ given by the commutative diagram

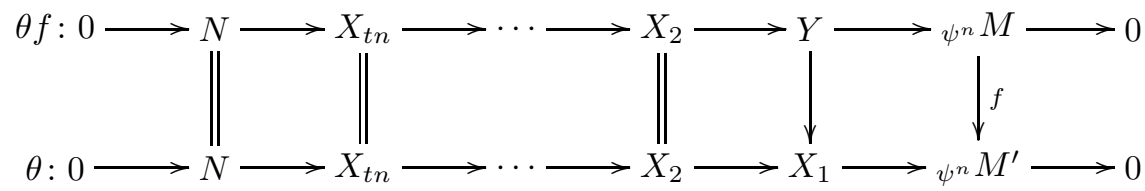

in which the module $Y$ is a pullback. For a homogeneous element $\eta \in H^{t m}$ we then get

$$
\begin{aligned}
\hat{f}(\theta \cdot \eta) & =(-1)^{|\eta||\theta|} \hat{f}(\eta \cdot \theta) \\
& =(-1)^{|\eta||\theta|} \hat{f}\left(\left(\eta \otimes_{\Lambda} N\right) \circ_{\psi^{m}} \theta\right) \\
& =(-1)^{|\eta||\theta|}\left(\eta \otimes_{\Lambda} N\right) \circ_{\psi^{m}}(\theta f) \\
& =(-1)^{|\eta||\theta|} \eta \cdot \hat{f}(\theta) \\
& =\hat{f}(\theta) \cdot \eta,
\end{aligned}
$$

showing $\hat{f}$ is a homomorphism of $H$-modules. Similarly $\operatorname{Ext}_{\Lambda}^{t *}\left(\psi^{*}-,-\right)$ preserves maps in the second argument.

Remark. It should also be noted that in many cases considering only strongly commutative algebras is not a severe restriction. For example, let $H$ be a commutative Noetherian graded subalgebra of $\operatorname{HH}^{t *}\left({ }_{\psi^{*}} \Lambda_{1}, \Lambda\right)$, as will be the case in most of the results in this section. Then by $\overline{\mathrm{Be} 1}$, Proposition 2.1] there exists an integer $w$ and a homogeneous element $\eta \in H$ of positive degree, say $|\eta|=t n$, such that the multiplication map

$$
H^{t i} \stackrel{\eta \cdot}{\longrightarrow} H^{t(i+n)}
$$

is injective for $i \geq w$. Now for any homogeneous element $\theta \in H^{t m}$ the product $\theta \cdot \eta$ is by definition the extension $\theta \circ \psi^{m} \eta=\left(\theta \otimes_{\Lambda} \Lambda\right) \circ\left(\psi^{m} \Lambda_{1} \otimes_{\Lambda} \eta\right)$, which by the Yoneda relation (I7) from Section 3 equals the extension $(-1)^{|\eta||\theta|}\left(\Lambda \otimes_{\Lambda} \eta\right) \circ\left(\theta \otimes_{\Lambda} \psi^{n} \Lambda_{1}\right)=$ $(-1)^{|\eta||\theta|} \eta \circ \theta_{\psi^{-n}}$. However, the extension $\eta \circ \theta_{\psi^{-n}}$ corresponds to the product $\eta \cdot \psi_{\psi^{-n}} \theta_{\psi^{-n}}$ in $H$, and so since $H$ is commutative we get

$$
\eta \cdot \theta=\theta \cdot \eta=(-1)^{|\eta||\theta|} \eta \cdot \psi^{-n} \theta_{\psi^{-n}} .
$$

Now if $|\theta| \geq t w$ then $\theta=(-1)^{|\eta||\theta|} \psi_{\psi^{-n}} \theta_{\psi^{-n}}$ due to the injectivity of the multiplication map induced by $\eta$, and twisting both sides in this equality by $\psi^{n}$ from the left we get $\psi^{n} \theta=(-1)^{|\eta||\theta|} \theta_{\psi^{-n}}$.

The algebra $H$, being Noetherian, is generated over $H^{0}$ by a finite set of homogeneous elements, say $\left\{x_{0}, \ldots, x_{r}\right\}$. Suppose now that $k$ contains an infinite field. Then from the proof of [Be1, Theorem 2.5] we see that we may choose the element $\eta$ above such that $|\eta|=\operatorname{lcm}\left\{\left|x_{0}\right|, \ldots,\left|x_{r}\right|\right\}$ In particular, if all the generators $x_{0}, \ldots, x_{r}$ are of the same degree, i.e. $\left|x_{i}\right|=t$, then the subalgebra $H^{0} \oplus \bigoplus_{i=w}^{\infty} H^{t i}$ of $H$ is strongly commutative.

Having made all the necessary assumptions, we are now ready to define twisted support varieties. For two $\Lambda$-modules $X$ and $Y$, denote by $\mathrm{A}_{H}^{\psi}(X, Y)$ the ideal $\operatorname{Ann}_{H} \operatorname{Ext}_{\Lambda}^{t *}\left(\psi^{*} X, Y\right)$, i.e. the annihilator in $H$ of $\operatorname{Ext}_{\Lambda}^{t *}\left(\psi^{*} X, Y\right)$. This ideal is graded since $\operatorname{Ext}_{\Lambda}^{t *}\left(\psi^{*} X, Y\right)$ is a graded $H$-module. As for ordinary support varieties, the defining ideal for the twisted support variety of $M$ is $\mathrm{A}_{H}^{\psi}(M, \Lambda / \mathfrak{r})$.

Definition 4.1. The twisted support variety $\mathrm{V}_{H}^{\psi}(M)$ of $M$ (with respect to the automorphism $\psi$, the integer $t$ and the ring $H$ ) is the subset

$$
\mathrm{V}_{H}^{\psi}(M) \stackrel{\text { def }}{=}\left\{\mathfrak{m} \in \operatorname{MaxSpec} H \mid \mathrm{A}_{H}^{\psi}(M, \Lambda / \mathfrak{r}) \subseteq \mathfrak{m}\right\}
$$

of the maximal ideal spectrum of $H$. 
Note that since $M$ and therefore also $\operatorname{Ext}_{\Lambda}^{t *}\left(\psi^{*} M, \Lambda / \mathfrak{r}\right)$ is nonzero, the degree zero part of $\mathrm{A}_{H}^{\psi}(M, \Lambda / \mathfrak{r})$ must be contained in the radical rad $H^{0}$ of the local ring $H^{0}=\mathrm{Z}(\Lambda)$ (if not then $\mathrm{A}_{H}^{\psi}(M, \Lambda / \mathfrak{r})$ contains the identity in $H$ ). Hence the twisted variety $\mathrm{V}_{H}^{\psi}(M)$ always contains the unique graded maximal ideal

$$
\mathfrak{m}_{H}=\operatorname{rad} H^{0} \oplus H^{t} \oplus H^{2 t} \oplus \cdots
$$

of $H$, and we shall say the variety is trivial if it does not contain any other element.

The following proposition lists some elementary and more or less expected properties of twisted varieties.

Proposition 4.2. (i) The ideal $\mathrm{A}_{H}^{\psi}(M, M)$ is also a defining ideal for the twisted variety of $M$, i.e.

$$
\mathrm{V}_{H}^{\psi}(M)=\left\{\mathfrak{m} \in \operatorname{MaxSpec} H \mid \mathrm{A}_{H}^{\psi}(M, M) \subseteq \mathfrak{m}\right\} .
$$

(ii) If $\operatorname{Ext}_{\Lambda}^{t n}\left(\psi_{\psi^{n}} M, M\right)=0$ for $n \gg 0$, then $\mathrm{V}_{H}^{\psi}(M)$ is trivial. In particular, the twisted variety of $M$ is trivial whenever the projective or injective dimension of $M$ is finite.

(iii) Given nonzero $\Lambda$-modules $M^{\prime}$ and $M^{\prime \prime}$ and an exact sequence

$$
0 \rightarrow M^{\prime} \rightarrow M \rightarrow M^{\prime \prime} \rightarrow 0,
$$

the relation

$$
\mathrm{V}_{H}^{\psi}(M) \subseteq \mathrm{V}_{H}^{\psi}\left(M^{\prime}\right) \cup \mathrm{V}_{H}^{\psi}\left(M^{\prime \prime}\right)
$$

holds. Moreover, if $\Omega_{\Lambda}^{t-1}\left(M^{\prime}\right) \neq 0$, then the relation

$$
\mathrm{V}_{H}^{\psi}\left(M^{\prime \prime}\right) \subseteq \mathrm{V}_{H}^{\psi}(M) \cup \mathrm{V}_{H}^{\psi}\left(\Omega_{\Lambda}^{t-1}\left({ }_{\psi} M^{\prime}\right)\right)
$$

holds, and if $M^{\prime \prime}=\Omega_{\Lambda}^{t-1}\left(M^{\prime \prime \prime}\right)$ for some module $M^{\prime \prime \prime}$, then the relation

$$
\mathrm{V}_{H}^{\psi}\left(M^{\prime}\right) \subseteq \mathrm{V}_{H}^{\psi}(M) \cup \mathrm{V}_{H}^{\psi}\left(\psi^{-1} M^{\prime \prime \prime}\right)
$$

holds.

(iv) If $M^{\prime}$ and $M^{\prime \prime}$ are nonzero $\Lambda$-modules such that $M=M^{\prime} \oplus M^{\prime \prime}$, then $\mathrm{V}_{H}^{\psi}(M)=\mathrm{V}_{H}^{\psi}\left(M^{\prime}\right) \cup \mathrm{V}_{H}^{\psi}\left(M^{\prime \prime}\right)$.

Proof. For a $\Lambda$-module $N$, denote by $\mathrm{V}_{H}^{\psi}(M, N)$ the variety whose defining ideal is $\mathrm{A}_{H}^{\psi}(M, N)$. To prove (i), we must show that $\mathrm{V}_{H}^{\psi}(M, \Lambda / \mathfrak{r})$ and $\mathrm{V}_{H}^{\psi}(M, M)$ are equal, and we start by proving by induction on the length of $N$ that $\mathrm{V}_{H}^{\psi}(M, N)$ is contained in $\mathrm{V}_{H}^{\psi}(M, \Lambda / \mathfrak{r})$. For any simple $\Lambda$-module $S$ the ideal $\mathrm{A}_{H}^{\psi}(M, \Lambda / \mathfrak{r})$ is contained in $\mathrm{A}_{H}^{\psi}(M, S)$ since $S$ is a summand of $\Lambda / \mathfrak{r}$, hence $\mathrm{V}_{H}^{\psi}(M, S) \subseteq \mathrm{V}_{H}^{\psi}(M, \Lambda / \mathfrak{r})$. If the length of $N$ is greater than 2, take any simple submodule $S \subset N$ and consider the exact sequence

$$
0 \rightarrow S \rightarrow N \rightarrow N / S \rightarrow 0
$$

This sequence induces the exact sequence

$$
\operatorname{Ext}_{\Lambda}^{t *}\left(\psi_{*}^{*} M, S\right) \rightarrow \operatorname{Ext}_{\Lambda}^{t *}\left(\psi^{*} M, N\right) \rightarrow \operatorname{Ext}_{\Lambda}^{t *}\left(\psi_{*}^{*} M, N / S\right)
$$

of $H$-modules, from which the inclusion

$$
\mathrm{A}_{H}^{\psi}(M, S) \cdot \mathrm{A}_{H}^{\psi}(M, N / S) \subseteq \mathrm{A}_{H}^{\psi}(M, N)
$$

follows. This implies that $\mathrm{V}_{H}^{\psi}(M, N) \subseteq \mathrm{V}_{H}^{\psi}(M, S) \cup \mathrm{V}_{H}^{\psi}(M, N / S)$, and since both $\mathrm{V}_{H}^{\psi}(M, S)$ and $\mathrm{V}_{H}^{\psi}(M, N / S)$ are contained in $\mathrm{V}_{H}^{\psi}(M, \Lambda / \mathfrak{r})$ by induction, we get $\mathrm{V}_{H}^{\psi}(M, N) \subseteq \mathrm{V}_{H}^{\psi}(M, \Lambda / \mathfrak{r})$. In particular $\mathrm{V}_{H}^{\psi}(M, M)$ is contained in $\mathrm{V}_{H}^{\psi}(M, \Lambda / \mathfrak{r})$. The reverse inclusion $\mathrm{V}_{H}^{\psi}(M, \Lambda / \mathfrak{r}) \subseteq \mathrm{V}_{H}^{\psi}(M, M)$ follows from the inclusion $\mathrm{A}_{H}^{\psi}(M, M) \subseteq \mathrm{A}_{H}^{\psi}(M, \Lambda / \mathfrak{r})$ of ideals in $H$, thus proving (i).

Now suppose $\operatorname{Ext}_{\Lambda}^{t n}\left(\psi_{\psi^{n}} M, M\right)=0$ for $n \gg 0$, let $\mathfrak{m} \subset H$ be a maximal ideal containing $\mathrm{A}_{H}^{\psi}(M, \Lambda / \mathfrak{r})$, and let $x \in H^{+}=\bigoplus_{n=1}^{\infty} H^{t n}$ be a homogeneous element. 
As the scalar action from $H$ on $\operatorname{Ext}_{\Lambda}^{t *}\left(\psi^{*} M, \Lambda / \mathfrak{r}\right)$ factors through $\operatorname{Ext}_{\Lambda}^{t *}\left(\psi^{*} M, M\right)$, some power of $x$ must lie in $\mathrm{A}_{H}^{\psi}(M, \Lambda / \mathfrak{r})$. Therefore $x$ must be an element of $\mathfrak{m}$, implying $\mathfrak{m}$ is a graded ideal. But $\mathfrak{m}_{H}$ is the only graded maximal ideal of $H$. This proves (ii).

Next suppose we are given an exact sequence as in (iii). The sequence induces the exact sequence

$$
\operatorname{Ext}_{\Lambda}^{t *}\left(\psi^{*} M^{\prime \prime}, \Lambda / \mathfrak{r}\right) \rightarrow \operatorname{Ext}_{\Lambda}^{t *}\left(\psi^{*} M, \Lambda / \mathfrak{r}\right) \rightarrow \operatorname{Ext}_{\Lambda}^{t *}\left(\psi^{*} M^{\prime}, \Lambda / \mathfrak{r}\right)
$$

of $H$-modules, giving the inclusion

$$
\mathrm{A}_{H}^{\psi}\left(M^{\prime}, \Lambda / \mathfrak{r}\right) \cdot \mathrm{A}_{H}^{\psi}\left(M^{\prime \prime}, \Lambda / \mathfrak{r}\right) \subseteq \mathrm{A}_{H}^{\psi}(M, \Lambda / \mathfrak{r})
$$

of ideals of $H$. The relation $\mathrm{V}_{H}^{\psi}(M) \subseteq \mathrm{V}_{H}^{\psi}\left(M^{\prime}\right) \cup \mathrm{V}_{H}^{\psi}\left(M^{\prime \prime}\right)$ now follows.

The original exact sequence also induces the two exact sequences

$$
\begin{gathered}
0 \rightarrow \operatorname{Hom}_{\Lambda}\left(M^{\prime \prime}, \Lambda / \mathfrak{r}\right) \rightarrow \operatorname{Hom}_{\Lambda}(M, \Lambda / \mathfrak{r}) \\
\operatorname{Ext}_{\Lambda}^{t n-1}\left(\psi^{n} M^{\prime}, \Lambda / \mathfrak{r}\right) \rightarrow \operatorname{Ext}_{\Lambda}^{t n}\left(\psi^{n} M^{\prime \prime}, \Lambda / \mathfrak{r}\right) \rightarrow \operatorname{Ext}_{\Lambda}^{t n}\left(\psi^{n} M, \Lambda / \mathfrak{r}\right)
\end{gathered}
$$

for $n \geq 1$, in which we may identify $\operatorname{Ext}_{\Lambda}^{t n-1}\left(\psi_{\psi^{n}} M^{\prime}, \Lambda / \mathfrak{r}\right)$ with $\operatorname{Ext}_{\Lambda}^{t(n-1)}\left({ }_{\psi^{n-1}} \Omega_{\Lambda}^{t-1}\left({ }_{\psi} M^{\prime}\right), \Lambda / \mathfrak{r}\right)$. Consequently the inclusion

$$
\mathrm{A}_{H}^{\psi}(M, \Lambda / \mathfrak{r}) \cdot \mathrm{A}_{H}^{\psi}\left(\Omega_{\Lambda}^{t-1}\left({ }_{\psi} M^{\prime}\right), \Lambda / \mathfrak{r}\right) \subseteq \mathrm{A}_{H}^{\psi}\left(M^{\prime \prime}, \Lambda / \mathfrak{r}\right)
$$

holds whenever $\Omega_{\Lambda}^{t-1}\left(M^{\prime}\right) \neq 0$, giving $\mathrm{V}_{H}^{\psi}\left(M^{\prime \prime}\right) \subseteq \mathrm{V}_{H}^{\psi}(M) \cup \mathrm{V}_{H}^{\psi}\left(\Omega_{\Lambda}^{t-1}\left({ }_{\psi} M^{\prime}\right)\right)$.

Finally, the short exact sequence induces the exact sequence

$$
\operatorname{Ext}_{\Lambda}^{t n}\left(\psi^{n} M, \Lambda / \mathfrak{r}\right) \rightarrow \operatorname{Ext}_{\Lambda}^{t n}\left(\psi_{\psi^{n}} M^{\prime}, \Lambda / \mathfrak{r}\right) \rightarrow \operatorname{Ext}_{\Lambda}^{t n+1}\left(\psi^{n} M^{\prime \prime}, \Lambda / \mathfrak{r}\right)
$$

for $n \geq 0$. If $M^{\prime \prime}=\Omega_{\Lambda}^{t-1}\left(M^{\prime \prime \prime}\right)$ for some module $M^{\prime \prime \prime}$, then we may identify $\operatorname{Ext}_{\Lambda}^{t n+1}\left(\psi_{\psi^{n}} M^{\prime \prime}, \Lambda / \mathfrak{r}\right)$ with $\operatorname{Ext}_{\Lambda}^{t(n+1)}\left({ }_{\psi^{n+1}}\left({ }_{\psi^{-1}} M^{\prime \prime \prime}\right), \Lambda / \mathfrak{r}\right)$, and the inclusion

$$
\mathrm{A}_{H}^{\psi}(M, \Lambda / \mathfrak{r}) \cdot \mathrm{A}_{H}^{\psi}\left(\psi^{-1} M^{\prime \prime \prime}, \Lambda / \mathfrak{r}\right) \subseteq \mathrm{A}_{H}^{\psi}\left(M^{\prime}, \Lambda / \mathfrak{r}\right)
$$

holds. This gives $\mathrm{V}_{H}^{\psi}\left(M^{\prime}\right) \subseteq \mathrm{V}_{H}^{\psi}(M) \cup \mathrm{V}_{H}^{\psi}\left(\psi_{\psi^{-1}} M^{\prime \prime \prime}\right)$, and the proof of (iii) is complete.

To prove (iv), note that by (iii) the inclusion $\mathrm{V}_{H}^{\psi}(M) \subseteq \mathrm{V}_{H}^{\psi}\left(M^{\prime}\right) \cup \mathrm{V}_{H}^{\psi}\left(M^{\prime \prime}\right)$ holds, whereas the inclusions $\mathrm{A}_{H}^{\psi}(M, \Lambda / \mathfrak{r}) \subseteq \mathrm{A}_{H}^{\psi}\left(M^{\prime}, \Lambda / \mathfrak{r}\right)$ and $\mathrm{A}_{H}^{\psi}(M, \Lambda / \mathfrak{r}) \subseteq$ $\mathrm{A}_{H}^{\psi}\left(M^{\prime \prime}, \Lambda / \mathfrak{r}\right)$ give $\mathrm{V}_{H}^{\psi}\left(M^{\prime}\right) \subseteq \mathrm{V}_{H}^{\psi}(M)$ and $\mathrm{V}_{H}^{\psi}\left(M^{\prime \prime}\right) \subseteq \mathrm{V}_{H}^{\psi}(M)$.

As a corollary, we obtain a result whose analogue in the theory of support varieties says that varieties are invariant under the syzygy operator.

Corollary 4.3. If $\Omega_{\Lambda}^{t}(M)$ is nonzero, then $\mathrm{V}_{H}^{\psi}(M)=\mathrm{V}_{H}^{\psi}\left(\Omega_{\Lambda}^{t}\left({ }_{\psi} M\right)\right)$.

Proof. The exact sequence

$$
0 \rightarrow \Omega_{\Lambda}^{1}(M) \rightarrow P_{0} \rightarrow M \rightarrow 0
$$

gives $\mathrm{V}_{H}^{\psi}(M) \subseteq \mathrm{V}_{H}^{\psi}\left(P_{0}\right) \cup \mathrm{V}_{H}^{\psi}\left(\Omega_{\Lambda}^{t-1}\left({ }_{\psi} \Omega_{\Lambda}^{1}(M)\right)\right)$, and since $\mathrm{V}_{H}^{\psi}\left(P_{0}\right)$ is trivial we get $\mathrm{V}_{H}^{\psi}(M) \subseteq \mathrm{V}_{H}^{\psi}\left(\Omega_{\Lambda}^{t}\left({ }_{\psi} M\right)\right)$. On the other hand, the exact sequence

$$
0 \rightarrow \Omega_{\Lambda}^{t}\left({ }_{\psi} M\right) \rightarrow{ }_{\psi} P_{t-1} \rightarrow \Omega_{\Lambda}^{t-1}\left({ }_{\psi} M\right) \rightarrow 0
$$

gives $\mathrm{V}_{H}^{\psi}\left(\Omega_{\Lambda}^{t}\left({ }_{\psi} M\right)\right) \subseteq \mathrm{V}_{H}^{\psi}\left({ }_{\psi} P_{t-1}\right) \cup \mathrm{V}_{H}^{\psi}\left({ }_{\psi^{-1}}\left({ }_{\psi} M\right)\right)$, and since $\mathrm{V}_{H}^{\psi}\left({ }_{\psi} P_{t-1}\right)$ is trivial we get $\mathrm{V}_{H}^{\psi}\left(\Omega_{\Lambda}^{t}\left({ }_{\psi} M\right)\right) \subseteq \mathrm{V}_{H}^{\psi}(M)$.

We illustrate this last result with an example. 
Example. Suppose $k$ is a field and $\Lambda$ is a Frobenius algebra, let $\psi=\nu_{\Lambda}$ be the Nakayama automorphism of $\Lambda$, and take $t=2$. We saw in the example following Lemma 2.1 that the Auslander-Reiten translation $\tau=D \operatorname{Tr}$ is isomorphic to $\Omega_{\Lambda}^{2} \mathcal{N}$, where $\mathcal{N}$ is the Nakayama functor $D \operatorname{Hom}_{\Lambda}(-, \Lambda)$. Moreover, in the example prior to Proposition 3.1 we saw that the latter is isomorphic to ${ }_{\nu} \Lambda_{1} \otimes_{\Lambda}-$. Therefore, from the corollary above we get

$$
\mathrm{V}_{H}^{\nu}(M)=\mathrm{V}_{H}^{\nu}\left(\Omega_{\Lambda}^{2}\left({ }_{\nu} M\right)\right)=\mathrm{V}_{H}^{\nu}\left(\Omega_{\Lambda}^{2}\left({ }_{\nu} \Lambda_{1} \otimes_{\Lambda} M\right)\right)=\mathrm{V}_{H}^{\nu}(\tau(M))
$$

whenever $\Omega_{\Lambda}^{2}(M)$ is nonzero.

A fundamental feature within the theory of support varieties for modules over both group algebras of finite groups and complete intersections is the finite generation of $\operatorname{Ext}^{*}(X, Y)$ (where $X$ and $Y$ are modules over the ring in question) as a module over the commutative Noetherian graded ring of cohomological operators (see Ben] and $\mathrm{Car}$ for the group ring case and $\mathrm{Avr}$ and $\mathrm{AvB}$. for the complete intersection case). In order to obtain a similar theory for selfinjective algebras in EHSST, it was necessary to assume that the same finite generation hypothesis held, since there exist selfinjective algebras for which it does not hold.

We now introduce a finite generation hypothesis similar to that used in Be1, where instead of assuming finite generation of $\operatorname{Ext}^{*}(X, Y)$ for all modules $X$ and $Y$, a local variant focusing on a single module was used.

Assumption $(\mathbf{F g}(M, H, \psi, t))$. Given the automorphism $\Lambda \stackrel{\psi}{\rightarrow} \Lambda$ and the integer $t \in \mathbb{N}$, there exists a strongly commutative Noetherian graded subalgebra $H=$ $\bigoplus_{n=0}^{\infty} H^{t n}$ of $\operatorname{HH}^{t *}\left(\psi_{*} \Lambda_{1}, \Lambda\right)$ such that $H^{0}=\operatorname{HH}^{0}(\Lambda, \Lambda)=\mathrm{Z}(\Lambda)$, and with the property that $\operatorname{Ext}_{\Lambda}^{t *}\left(\psi^{*} M, \Lambda / \mathfrak{r}\right)$ is a finitely generated $H$-module.

As mentioned in the remark prior to Definition 4.1, considering only strongly commutative algebras instead of "ordinary" commutative algebras is not a severe restriction. In fact, the following result shows that if $\mathbf{F g}(M, H, \psi, t)$ holds for a commutative Noetherian graded algebra $H$ which is not necessarily strongly commutative, then there exist a positive integer $s$ and a strongly commutative Noetherian subalgebra $H^{\prime} \subseteq H$ for which $\mathbf{F g}\left(M, H^{\prime}, \psi^{s}, t s\right)$ holds.

Proposition 4.4. Let $\Lambda \stackrel{\psi}{\rightarrow} \Lambda$ be an automorphism and $t \in \mathbb{N}$ an integer, and suppose there exists a commutative Noetherian graded subalgebra $H=\bigoplus_{n=0}^{\infty} H^{t n}$ of $\mathrm{HH}^{t *}\left({ }_{\psi^{*}} \Lambda_{1}, \Lambda\right)$ such that $H^{0}=\mathrm{HH}^{0}(\Lambda, \Lambda)=\mathrm{Z}(\Lambda)$, and with the property that $\operatorname{Ext}_{\Lambda}^{t *}\left(\psi^{*} M, \Lambda / \mathfrak{r}\right)$ is a finitely generated $H$-module. Then there exist a positive integer $s$ and a strongly commutative Noetherian graded subalgebra $H^{\prime}$ of $H$ for which $\mathbf{F g}\left(M, H^{\prime}, \psi^{s}, t s\right)$ holds

Proof. From the remark prior to Definition 4.1 we see that there exist two positive integers $n$ and $w$ such that $\psi^{n} \theta=\theta_{\psi^{-n}}$ for every homogeneous element $\theta \in H$ with $|\theta| \geq t w$. Let $\eta_{1}, \ldots, \eta_{r} \in H$ be homogeneous elements of positive degrees generating $H$ as an algebra over $H^{0}$, and denote the integer $w n$ by $s$. Then the subalgebra $H^{\prime}=H^{0}\left[\eta_{1}^{s}, \ldots, \eta_{r}^{s}\right]$ of $H$ is strongly commutative with respect to the

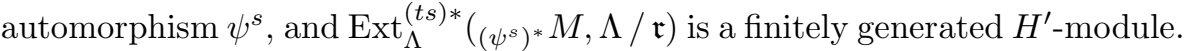

We now show that introducing the above finite generation hypothesis enables us to compute the dimension of the twisted variety of a module. Recall first that if $X=\bigoplus_{n=0}^{\infty} X_{n}$ is a graded $k$-module of finite type (that is, the $k$-length of $X_{n}$ is finite for all $n$ ), then the rate of growth of $X$, denoted $\gamma(X)$, is defined as

$$
\gamma(X) \stackrel{\text { def }}{=} \inf \left\{c \in \mathbb{N} \cup\{0\} \mid \exists a \in \mathbb{R} \text { such that } \ell_{k}\left(X_{n}\right) \leq a n^{c-1} \text { for } n \gg 0\right\} .
$$


For a $\Lambda$-module $Y$ with minimal projective resolution $\cdots \rightarrow Q_{1} \rightarrow Q_{0} \rightarrow Y \rightarrow 0$, we define the $m$-complexity of $Y$ (where $m \geq 1$ is a number) as the rate of growth of $\bigoplus_{n=0}^{\infty} Q_{m n}$, and denote it by $\mathrm{cx}^{m} Y$. Note that the 1-complexity of a module coincides with the usual notion of complexity, and that the identity

$$
\mathrm{cx}^{m} Y=\gamma\left(\operatorname{Ext}_{\Lambda}^{m *}(Y, \Lambda / \mathfrak{r})\right)
$$

always holds (the latter can be seen by adopting the arguments given in Be1, Section 3]).

The following result allows us to compute the dimension of $\mathrm{V}_{H}^{\psi}(M)$ in terms of the $t$-complexity of $M$ provided $\mathbf{F g}(M, H, \psi, t)$ is satisfied. In particular Dade's Lemma holds.

Proposition 4.5. If $\mathbf{F g}(M, H, \psi, t)$ holds, then $\operatorname{dim} \mathrm{V}_{H}^{\psi}(M)=\mathrm{cx}^{t} M$. In particular $\mathrm{V}_{H}^{\psi}(M)$ is trivial if and only if $M$ has finite projective dimension.

Proof. Adopting the arguments used to prove [Ben, Proposition 5.7.2] and [EHSST, Proposition 2.1] gives $\operatorname{dim} \mathrm{V}_{H}^{\psi}(M)=\gamma\left(\operatorname{Ext}_{\Lambda}^{t *}\left(\psi_{*}^{*} M, \Lambda / \mathfrak{r}\right)\right)$. For any $\Lambda$-module $Y$ and any $k$-automorphism $\Lambda \stackrel{\phi}{\rightarrow} \Lambda$ there is an isomorphism $Y \simeq{ }_{\phi} Y$ of $k$-modules, and so if $\cdots \rightarrow Q_{1} \rightarrow Q_{0} \rightarrow Y \rightarrow 0$ is a minimal projective resolution we see that the $m$-complexity of $Y$ equals the rate of growth of $\bigoplus_{n=0}^{\infty} \phi^{n}\left(Q_{m n}\right)$. In particular the equalities

$$
\mathrm{cx}^{t} M=\gamma\left(\bigoplus_{n=0}^{\infty} \psi^{n}\left(P_{t n}\right)\right)=\gamma\left(\operatorname{Ext}_{\Lambda}^{t *}\left(\psi^{*} M, \Lambda / \mathfrak{r}\right)\right)
$$

hold, where

$$
\mathbb{P}: \cdots \rightarrow P_{2} \stackrel{d_{2}}{\longrightarrow} P_{1} \stackrel{d_{1}}{\longrightarrow} P_{0} \stackrel{d_{0}}{\longrightarrow} M \rightarrow 0
$$

is the minimal projective resolution of $M$.

Note that whenever $\mathbf{F g}(M, H, \psi, t)$ is satisfied the dimension of $\mathrm{V}_{H}^{\psi}(M)$, and therefore also the $t$-complexity of $M$, must be finite; since $H$ is commutative graded Noetherian it is generated as an algebra over $H^{0}$ by a finite set $\left\{x_{0}, \ldots, x_{r}\right\}$ of homogeneous elements of positive degrees, giving $\gamma\left(\operatorname{Ext}_{\Lambda}^{t *}\left(\psi^{*} M, \Lambda / \mathfrak{r}\right)\right) \leq r$ (see the discussion prior to Be1, Proposition 3.1]). It then follows from the proof of the above proposition that $\mathrm{cx}^{t} M \leq r$.

The next result gives a sufficient and necessary condition for the variety to be one dimensional. Recall that a $\Lambda$-module $Y$ is periodic if there exists a positive integer $p$ such that $Y \simeq \Omega_{\Lambda}^{p}(Y)$, whereas it is eventually periodic if $\Omega_{\Lambda}^{i}(Y)$ is periodic for some $i \geq 0$. For a $k$-automorphism $\Lambda \stackrel{\phi}{\rightarrow} \Lambda$ we define $Y$ to be $\phi$-periodic if there exists a positive integer $p$ such that $Y \simeq \Omega_{\Lambda}^{p}\left({ }_{\phi} Y\right)$, and eventually $\phi$-periodic if $\Omega_{\Lambda}^{i}(Y)$ is $\phi$-periodic for some $i \geq 0$.

Proposition 4.6. If $\mathbf{F g}(M, H, \psi, t)$ holds, then $\operatorname{dim} \mathrm{V}_{H}^{\psi}(M)=1$ if and only if $M$ is eventually $\psi^{i}$-periodic for some $i \geq 1$. Moreover, when this occurs there is a

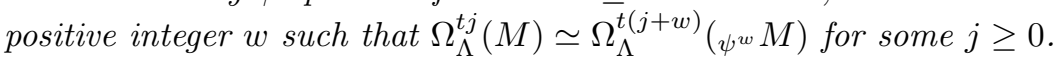

Proof. If $M$ is eventually $\psi^{i}$-periodic, then the sequence

$$
\ell_{k}\left(P_{0}\right), \ell_{k}\left(P_{1}\right), \ell_{k}\left(P_{2}\right), \ldots
$$

must be bounded, that is, the 1-complexity of $M$ is 1 . But then the sequence

$$
\ell_{k}\left(P_{0}\right), \ell_{k}\left(P_{t}\right), \ell_{k}\left(P_{2 t}\right), \ldots
$$

is also bounded, that is, the $t$-complexity of $M$ is also 1 , and consequently $\operatorname{dim} \mathrm{V}_{H}^{\psi}(M)=1$. 
Conversely, suppose the latter of the above sequences is bounded. By Be1, Proposition 2.1] there exists a homogeneous element $\eta \in H$ of positive degree, say $|\eta|=t w$, such that the multiplication map

$$
\operatorname{Ext}_{\Lambda}^{t i}\left(\psi^{i} M, \Lambda / \mathfrak{r}\right) \stackrel{\cdot \eta}{\rightarrow} \operatorname{Ext}_{\Lambda}^{t(i+w)}\left(\psi_{\psi^{i+w}} M, \Lambda / \mathfrak{r}\right)
$$

is injective for $i \gg 0$. Represent the element $\eta \otimes_{\Lambda} M \in \operatorname{Ext}_{\Lambda}^{t *}\left(\psi_{*}^{*} M, M\right)$ by a map $f_{\eta}: \Omega_{\Lambda}^{t w}\left({ }_{\psi_{w}} M\right) \rightarrow M$. Then for each $i \geq 0$ there is a map $f_{i}: \psi_{\psi^{w}}\left(P_{t w+i}\right) \rightarrow P_{i}$ making the diagram

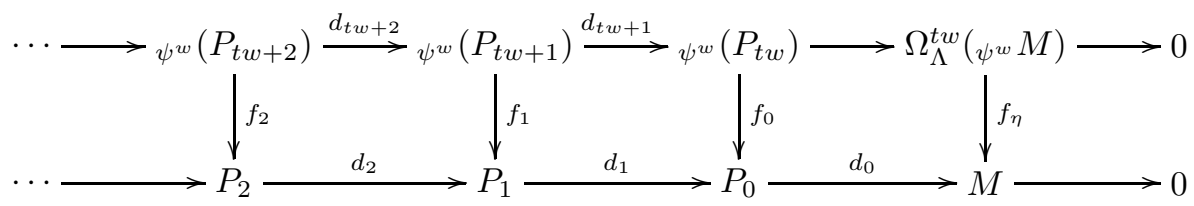

with exact rows commute. If $\theta \in \operatorname{Ext}_{\Lambda}^{t *}\left(\psi^{*} M, \Lambda / \mathfrak{r}\right)$ is a homogeneous element, say $|\theta|=t n$, and represented by a map $f_{\theta}: \psi^{n}\left(P_{t n}\right) \rightarrow \Lambda / \mathfrak{r}$, then $\theta \eta \in$ $\operatorname{Ext}_{\Lambda}^{t(w+n)}\left(_{\psi^{w+n}} M, \Lambda / \mathfrak{r}\right)$ is represented by the composite map

$$
\psi^{w+n}\left(P_{t(w+n)}\right) \stackrel{\psi^{n} f_{t n}}{\longrightarrow} \psi^{n}\left(P_{t n}\right) \stackrel{f_{\theta}}{\longrightarrow} \Lambda / \mathfrak{r} .
$$

For any $i \geq 0$ the complex $\psi_{\psi^{i}} \mathbb{P}$ is a minimal projective resolution of $\psi_{\psi^{i}} M$, and therefore $\operatorname{Ext}_{\Lambda}^{t i}\left(\psi_{\psi^{i}} M, \Lambda / \mathfrak{r}\right)=\operatorname{Hom}_{\Lambda}\left({ }_{\psi^{i}}\left(P_{t i}\right), \Lambda / \mathfrak{r}\right)$. Moreover, the multiplication map

$$
\operatorname{Ext}_{\Lambda}^{t i}\left(\psi_{\psi^{i}} M, \Lambda / \mathfrak{r}\right) \stackrel{\cdot \eta}{\longrightarrow} \operatorname{Ext}_{\Lambda}^{t(i+w)}\left(_{\psi^{i+w}} M, \Lambda / \mathfrak{r}\right)
$$

is just the map

$$
\begin{aligned}
& \operatorname{Hom}_{\Lambda}\left(\psi^{i}\left(P_{t i}\right), \Lambda / \mathfrak{r}\right) \stackrel{\left(\psi_{\psi^{i}} f_{t i}\right)^{*}}{\longrightarrow} \operatorname{Hom}_{\Lambda}\left(\psi^{i+w}\left(P_{t(i+w)}\right), \Lambda / \mathfrak{r}\right) \\
& g \quad \mapsto \quad g \circ \psi^{i} f_{t i},
\end{aligned}
$$

and since the exact sequence

$$
\psi^{i+w}\left(P_{t(i+w)}\right) \stackrel{\psi^{i} f_{t i}}{\longrightarrow} \psi^{i}\left(P_{t i}\right) \rightarrow \psi^{i}\left(\text { Coker } f_{t i}\right) \rightarrow 0
$$

shows that the kernel of the multiplication map is isomorphic to $\operatorname{Hom}_{\Lambda}\left(\psi_{\psi^{i}}\left(\operatorname{Coker} f_{t i}\right), \Lambda / \mathfrak{r}\right)$, we see that Coker $f_{t i}=0$ for $i \gg 0$. Consequently, for each $i \gg 0$ there exists a surjective map $\Omega_{\Lambda}^{t(w+i)}\left({ }_{\psi^{w}} M\right) \rightarrow \Omega_{\Lambda}^{t i}(M)$ and therefore also a sequence

$$
\left.\left.\cdots \rightarrow \Omega_{\Lambda}^{t(3 w+i)}{ }_{\psi^{3 w}} M\right) \rightarrow \Omega_{\Lambda}^{t(2 w+i)}{ }_{\psi^{2 w}} M\right) \rightarrow \Omega_{\Lambda}^{t(w+i)}{ }_{\left.\psi_{\psi^{w}} M\right)} M \Omega_{\Lambda}^{t i}(M)
$$

of surjections. However, by assumption the sequence

$$
\ell_{k}\left(P_{0}\right), \ell_{k}\left(P_{t}\right), \ell_{k}\left(P_{2 t}\right), \ldots
$$

is bounded, and therefore $\Omega_{\Lambda}^{t((q+1) w+i)}\left(_{\psi^{(q+1) w}} M\right)$ must be isomorphic to $\Omega_{\Lambda}^{t(q w+i)}{ }_{\left.\psi_{\psi^{q w}} M\right)}$ for large $q$ and $i$. By setting $j=q w+i$ and twisting with the automorphism $\psi^{-q w}$, we see that $\Omega_{\Lambda}^{t j}(M) \simeq \Omega_{\Lambda}^{t(j+w)}\left({ }_{\psi_{w}} M\right)$ for $j \gg 0$.

As a particular case of the proposition we obtain the following result on $D$ Trperiodicity over a Frobenius algebra.

Proposition 4.7. Suppose $k$ is a field and $\Lambda$ is a Frobenius algebra, and let $\Lambda \stackrel{\nu}{\rightarrow} \Lambda$ be a Nakayama automorphism. If $M$ does not have a nonzero projective summand and $\mathbf{F g}\left(M, H, \nu^{n}, 2 n\right)$ holds for some $n \geq 1$, then $\operatorname{dim} \mathrm{V}_{H}^{\nu}(M)=1$ if and only if $M \simeq \tau^{p}(M)$ for some $p \geq 1$. 
Proof. By the previous proposition the variety $\mathrm{V}_{H}^{\nu}(M)$ is one dimensional if and only if there is a positive integer $w$ such that $\Omega_{\Lambda}^{2 n j}(M) \simeq \Omega_{\Lambda}^{2 n(j+w)}\left({ }_{\nu^{n w}} M\right)$ for some $j \geq 0$. Taking cosyzygies we see that the latter happens precisely when $M \simeq \Omega_{\Lambda}^{2 n w}\left({ }_{\nu}^{n w} M\right)$, that is, when $M \simeq \tau^{n w}(M)$.

We illustrate this last result with an example.

Example. Let $k$ be an algebraically closed field of odd characteristic and $q \in k$ a nonzero element which is not a root of unity, and denote by $\Lambda$ the $k$-algebra

$$
\Lambda=k\langle x, y\rangle /\left(x^{2}, x y+q y x, y^{2}\right) .
$$

We saw in the example following Proposition 3.1 that the Nakayama automorphism $\nu$ of $\Lambda$ is defined by

$$
x \mapsto-q^{-1} x, \quad y \mapsto-q y,
$$

and that $\mathrm{HH}^{4 *}\left(\nu^{2 *} \Lambda_{1}, \Lambda\right)$ is isomorphic to the fibre product $k[\theta] \times{ }_{k} k$ with $\theta$ of degree 4. In particular $\mathrm{HH}^{4 *}\left({ }_{\nu}{ }_{2 *} \Lambda_{1}, \Lambda\right)$ is strongly commutative, and we denote this ring by $H$.

For elements $\alpha, \beta \in k$, denote by $M_{(\alpha, \beta)}$ the $\Lambda$-module $\Lambda(\alpha x+\beta y)$ (see Sma for a counterexample, using the module $M_{(1,1)}$, to a question raised by Auslander, a question for which a counterexample was first given in [JoS]). Consider the module $M=M_{(1, \beta)}$ for $\beta \neq 0$, and for each $i \geq 0$ let $P_{i}=\Lambda$. The sequence

$$
\mathbb{P}: \cdots \rightarrow P_{3} \stackrel{\cdot\left(x+q^{3} \beta y\right)}{\longrightarrow} P_{2} \stackrel{\cdot\left(x+q^{2} \beta y\right)}{\longrightarrow} P_{1} \stackrel{\cdot(x+q \beta y)}{\longrightarrow} P_{0} \stackrel{\cdot(x+\beta y)}{\longrightarrow} M \rightarrow 0
$$

is a minimal projective resolution of $M$, hence since ${ }_{\nu^{n}} \mathbb{P}$ is a minimal projective resolution of ${ }_{\nu^{n}} M$ for any $n \geq 1$ we see that $\operatorname{Ext}_{\Lambda}^{2 n}\left({ }_{\nu^{n}} M, k\right)=\operatorname{Hom}_{\Lambda}\left({ }_{\nu^{n}} \Lambda, k\right)$ is one dimensional. We shall prove that $\mathbf{F g}(M, H, \nu, 2)$ holds.

Recall from the example following Proposition 3.1 the minimal bimodule projective resolution

$$
(\mathbb{F}, \delta): \cdots \rightarrow F^{n+1} \stackrel{\delta}{\rightarrow} F^{n} \stackrel{\delta}{\rightarrow} F^{n-1} \rightarrow \cdots
$$

of $\Lambda$, where the set $\left\{\tilde{f}_{i}^{n}\right\}_{i=0}^{n}$ generates $F^{n}$ as a $\Lambda^{\mathrm{e}}$-module and the differential $\delta: F^{n} \rightarrow F^{n-1}$ is given by

$$
\tilde{f}_{i}^{n} \mapsto\left[x \tilde{f}_{i}^{n-1}+(-1)^{n} q^{i} \tilde{f}_{i}^{n-1} x\right]+\left[q^{n-i} y \tilde{f}_{i-1}^{n-1}+(-1)^{n} \tilde{f}_{i-1}^{n-1} y\right] .
$$

The element $\theta \in H^{4}$ represented by the map

$$
\begin{aligned}
g_{4}: \nu^{2} F_{1}^{4} & \rightarrow \Lambda \\
\tilde{f}_{i}^{4} & \mapsto \begin{cases}1 & \text { for } i=2 \\
0 & \text { for } i \neq 2\end{cases}
\end{aligned}
$$

generates $H$ as an algebra over $H^{0}$. The resolution $\mathbb{F} \otimes_{\Lambda} M$ is also a projective resolution of $M$, and defining $\Lambda$-linear maps

$$
\begin{aligned}
h_{n}: P_{n} & \rightarrow F^{n} \otimes_{\Lambda} M \\
1 & \mapsto\left(\sum_{i=0}^{n} q^{\frac{i(i+1)}{2}} \beta^{i} \tilde{f}_{i}^{n}\right) \otimes(x+\beta y)
\end{aligned}
$$

gives a commutative diagram

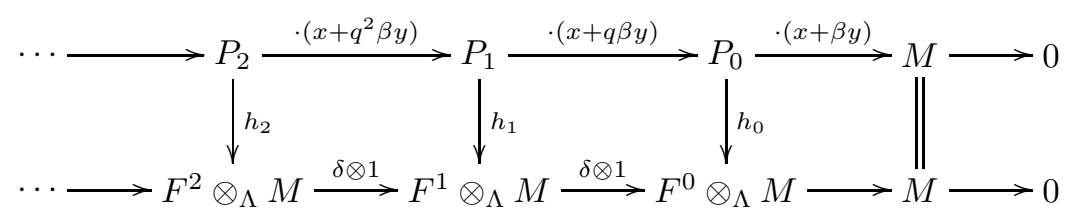


with exact rows. Consequently, the element $\theta \otimes_{\Lambda} M \in \operatorname{Ext}_{\Lambda}^{4}\left(\nu^{2} M, M\right)$ is represented by the composite map

$$
\begin{aligned}
& \bar{f}_{\theta}: \nu_{\nu^{2}}\left(P_{4}\right) \stackrel{\left(g_{4} \otimes 1\right) \circ h_{4}}{\longrightarrow} \Lambda \otimes_{\Lambda} M \simeq M \\
& 1 \mapsto \quad q^{3} \beta^{2}(x+\beta y) \text {. }
\end{aligned}
$$

Now for each $i \geq 0$ define $f_{i}: \nu^{2}\left(P_{i+4}\right) \rightarrow_{\nu^{2}}\left(P_{i}\right)$ by $1 \mapsto q^{2 i+3} \beta^{2}$. We then obtain a commutative diagram

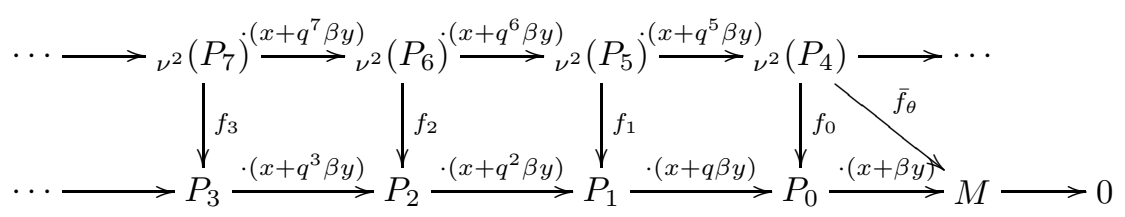

with exact rows, hence if $\mu \in \operatorname{Ext}_{\Lambda}^{2 n}\left(\nu^{n} M, k\right)=\operatorname{Hom}_{\Lambda}\left(\nu_{\nu^{n}}\left(P_{2 n}\right), k\right)$ is an element represented by a map $\bar{f}_{\mu}: \nu^{n}\left(P_{2 n}\right) \rightarrow k$ we see that the element $\mu \cdot \theta \in$ $\operatorname{Ext}_{\Lambda}^{2(n+2)}\left({ }_{\nu(n+2)} M, k\right)$ is represented by the composite map

$$
\nu^{(n+2)}\left(P_{2(n+2)}\right) \stackrel{\nu^{n}\left(f_{2 n}\right)}{\longrightarrow} \nu^{n}\left(P_{2 n}\right) \stackrel{\bar{f}_{\mu}}{\longrightarrow} k .
$$

Moreover, this composition is nonzero whenever $\mu$ is nonzero, since $f_{2 n}$ is just multiplication with $q^{4 n+3} \beta^{2}$. Therefore, since $\operatorname{Ext}_{\Lambda}^{2 n}\left(\nu^{n} M, k\right)$ is one dimensional for each $n \geq 1$, the $H$-module $\operatorname{Ext}_{\Lambda}^{2 *}\left(\nu^{*} M, k\right)$ is finitely generated; it is generated as an $H$-module by any $k$-basis in $\operatorname{Ext}_{\Lambda}^{0}\left({ }_{\nu^{0}} M, k\right)=\operatorname{Hom}_{\Lambda}(M, k)$ together with any nonzero elements $\mu_{1} \in \operatorname{Ext}_{\Lambda}^{2}\left({ }_{\nu} M, k\right)$ and $\mu_{2} \in \operatorname{Ext}_{\Lambda}^{4}\left(\nu^{2} M, k\right)$.

The above shows that $\operatorname{Fg}\left(M_{(1, \beta)}, H, \nu, 2\right)$ holds for $\beta \neq 0$, and since the 2complexity of $M_{(1, \beta)}$ is obviously 1 we see from Proposition 4.5 that the variety $\mathrm{V}_{H}^{\nu}\left(M_{(1, \beta)}\right)$ is one dimensional. From Proposition 4.7 we conclude that $M_{(1, \beta)} \simeq$ $\tau^{w}\left(M_{(1, \beta)}\right)$ for some $w \geq 1$. Indeed, in [LiS] it is shown that $M_{(1, \beta)}$ is isomorphic to $\tau\left(M_{(1, \beta)}\right)$.

Recall that each nonprojective indecomposable $\Lambda$-module is annihilated by $y x$, and is therefore a module over the algebra $\Lambda /(y x)$ (see [Sch, Section 4]). The latter is stably equivalent to the Kronecker algebra, an equivalence under which the representation

$$
k \stackrel{\beta}{\underset{\alpha}{\longrightarrow}} k
$$

corresponds to the module $M_{(\alpha, \beta)}$. Denoting $M_{(\alpha, \beta)}$ by $M_{(\alpha, \beta)}^{1}$, it follows from the well known representation theory of the Kronecker algebra (see, for example, ARS and ASS] that the indecomposable $\tau$-periodic $\Lambda$-modules are divided into distinct countable classes $\left\{M_{(\alpha, \beta)}^{i}\right\}_{i=1}^{\infty}$, one for each pair $(\alpha, \beta) \in\{(0,1)\} \cup\{(1, \beta)\}_{\beta \in k}$, such that for each $i \geq 1$ there exists a short exact sequence

$$
0 \rightarrow M_{(\alpha, \beta)}^{i} \rightarrow M_{(\alpha, \beta)}^{i-1} \oplus M_{(\alpha, \beta)}^{i+1} \rightarrow M_{(\alpha, \beta)}^{i} \rightarrow 0,
$$

where $M_{(\alpha, \beta)}^{0}=0$. Now each such exact sequence induces an exact sequence

$$
\operatorname{Ext}_{\Lambda}^{2 *}\left(\nu^{*} M_{\alpha}^{i}, k\right) \rightarrow \operatorname{Ext}_{\Lambda}^{2 *}\left(\nu^{*} M_{\alpha}^{i-1}, k\right) \oplus \operatorname{Ext}_{\Lambda}^{2 *}\left(\nu^{*} M_{\alpha}^{i+1}, k\right) \rightarrow \operatorname{Ext}_{\Lambda}^{2 *}\left(\nu^{*} M_{\alpha}^{i}, k\right)
$$

of $H$-modules, and so since $H$ is Noetherian and $\operatorname{Ext}_{\Lambda}^{2 *}\left(\nu_{\nu^{*}} M_{(1, \beta)}^{1}, k\right)$ is a finitely generated $H$-module whenever $\beta$ is nonzero, an induction argument shows that $\operatorname{Ext}_{\Lambda}^{2 *}\left(\nu^{*} M_{(1, \beta)}^{i}, k\right)$ is a finitely generated $H$-module for any $i \geq 1$ and $\beta \neq 0$. We conclude that $\mathbf{F g}\left(M_{(1, \beta)}^{i}, H, \nu, 2\right)$ holds for all the modules $\left\{M_{(1, \beta)}^{i}\right\}_{i=1}^{\infty}$ when $\beta$ is nonzero. 
However, there are two more classes of indecomposable $\tau$-periodic $\Lambda$-modules, namely $\left\{M_{(1,0)}^{i}\right\}_{i=0}^{\infty}$ and $\left\{M_{(0,1)}^{i}\right\}_{i=0}^{\infty}$. Do these modules satisfy the finite generation hypothesis? The answer is no, and to see this, consider the module $M_{(1,0)}=\Lambda x$. Letting $P_{i}=\Lambda$ for each $i \geq 0$ we see that the sequence

$$
\cdots \rightarrow P_{3} \stackrel{\cdot x}{\rightarrow} P_{2} \stackrel{\cdot x}{\rightarrow} P_{1} \stackrel{\cdot x}{\rightarrow} P_{0} \stackrel{\cdot x}{\rightarrow} M_{(1,0)} \rightarrow 0
$$

is a minimal projective resolution of $M_{(1,0)}$, and defining $\Lambda$-linear maps

$$
\begin{aligned}
h_{n}: P_{n} & \rightarrow F^{n} \otimes_{\Lambda} M_{(1,0)} \\
1 & \mapsto \tilde{f}_{0}^{n} \otimes x
\end{aligned}
$$

gives a commutative diagram

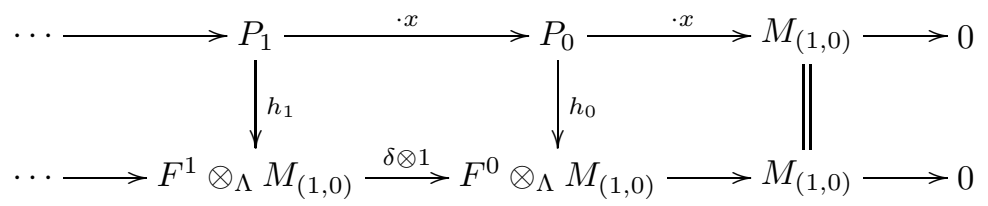

with exact rows. Since the map $h_{4}$ does not "hit" the generator $\tilde{f}_{2}^{4} \otimes x \in F^{4} \otimes_{\Lambda}$ $M_{(1,0)}$, we see that the element $\theta \otimes_{\Lambda} M \in \operatorname{Ext}_{\Lambda}^{4}\left(\nu^{2} M, M\right)$ is represented by the zero map. This shows that $\operatorname{Ext}_{\Lambda}^{2 *}\left(\nu^{*} M_{(1,0)}^{i}, k\right)$ cannot be a finitely generated $H$-module, and a similar argument shows that the same is true for the module $M_{(0,1)}$.

Note also that the finite generation condition cannot hold for any nonzero indecomposable nonprojective $\Lambda$-module which is not $\tau$-periodic; if $X$ is such a module and $\operatorname{Ext}_{\Lambda}^{2 *}\left(\nu^{*} X, k\right)$ is finitely generated over $H$, then the rate of growth of $\operatorname{Ext}_{\Lambda}^{2 *}\left(\nu^{*} X, k\right)$ is not more than that of $H$. However, the latter equals the Krull dimension of $H$, thus $\gamma\left(\operatorname{Ext}_{\Lambda}^{2 *}\left(\nu^{*} X, k\right)\right) \leq \gamma(H)=1$. Since $X$ is nonprojective we conclude that the rate of growth of $\operatorname{Ext}_{\Lambda}^{2 *}\left(\nu^{*} X, k\right)$ is 1 , and so by Proposition 4.5 the variety $\mathrm{V}_{H}^{\nu}(X)$ is one dimensional. But then Proposition 4.7 implies $X$ is $\tau$-periodic, a contradiction.

Returning to the general theory, we now impose the finite generation hypothesis on both $M$ and $\Omega_{\Lambda}^{1}(M)$. The following result shows that, in this situation, if the variety of $M$ is nontrivial (that is, when $M$ does not have finite projective dimension) then there exists a homogeneous element in $H$ "cutting down" the variety by one dimension. Recall first that if $\eta \in \mathrm{HH}^{t *}\left(\psi_{\psi^{*}} \Lambda_{1}, \Lambda\right)$ is a homogeneous element, say $\eta \in \operatorname{Ext}_{\Lambda^{\mathrm{e}}}^{t m}\left(\psi^{m} \Lambda_{1}, \Lambda\right)$, then it can be represented by a $\Lambda^{\mathrm{e}}$-linear map $f_{\eta}: \Omega_{\Lambda^{\mathrm{e}}}^{t m}\left(\psi^{m} \Lambda_{1}\right) \rightarrow \Lambda$. This map yields a commutative diagram

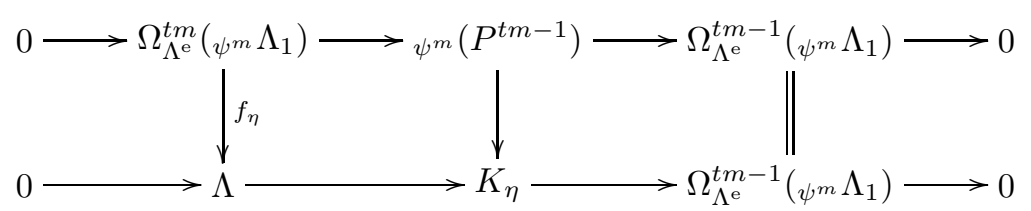

with exact rows, in which we have denoted by $P^{i}$ the $i$ th module in the minimal projective bimodule resolution of $\Lambda$. Note that up to isomorphism the module $K_{\eta}$ is independent of the map $f_{\eta}$ chosen to represent $\eta$.

Proposition 4.8. If both $\mathbf{F g}(M, H, \psi, t)$ and $\mathbf{F g}\left(\Omega_{\Lambda}^{1}(M), H, \psi, t\right)$ hold and $M$ does not have finite projective dimension, then there exists a homogeneous element $\eta \in H$ of positive degree such that $\operatorname{dim} \mathrm{V}_{H}^{\psi}\left(\Omega_{\Lambda^{\mathrm{e}}}^{1}\left(K_{\eta}\right) \otimes_{\Lambda} M\right)=\operatorname{dim} \mathrm{V}_{H}^{\psi}(M)-1$.

Proof. By assumption the $H$-modules $\operatorname{Ext}_{\Lambda}^{t *}\left(\psi_{*}^{*} M, \Lambda / \mathfrak{r}\right)$ and $\operatorname{Ext}_{\Lambda}^{t *}\left(\psi_{\psi^{*}} \Omega_{\Lambda}^{1}(M), \Lambda / \mathfrak{r}\right)$ are finitely generated, hence by slightly generalizing the proof of [Be1, Proposition 
2.1] we see that there exists a homogeneous element $\eta \in H$ of positive degree, say $\eta \in H^{t m} \subseteq \operatorname{Ext}_{\Lambda^{\mathrm{e}}}^{t m}\left(\psi^{m} \Lambda_{1}, \Lambda\right)$, such that the multiplication maps

$$
\begin{array}{rll}
\operatorname{Ext}_{\Lambda}^{t i}\left(\psi_{\psi^{i}} M, \Lambda / \mathfrak{r}\right) & \stackrel{\cdot \eta}{\rightarrow} & \operatorname{Ext}_{\Lambda}^{t(i+m)}\left(\psi_{\psi^{i+m}} M, \Lambda / \mathfrak{r}\right) \\
\operatorname{Ext}_{\Lambda}^{t i}\left({ }_{\psi^{i}} \Omega_{\Lambda}^{1}(M), \Lambda / \mathfrak{r}\right) & \left.\stackrel{\cdot \eta}{\longrightarrow} \operatorname{Ext}_{\Lambda}^{t(i+m)}{\left(\psi^{i+m}\right.}^{(i+1} \Omega_{\Lambda}^{1}(M), \Lambda / \mathfrak{r}\right)
\end{array}
$$

are both $k$-monomorphisms for $i \gg 0$. Consider the short exact sequence

$$
0 \rightarrow \Lambda \rightarrow K_{\eta} \rightarrow \Omega_{\Lambda^{\mathrm{e}}}^{t m-1}\left({ }_{\psi^{m}} \Lambda_{1}\right) \rightarrow 0
$$

obtained from $\eta$. As $\Omega_{\Lambda^{\mathrm{e}}}^{t m-1}\left(\psi_{\psi^{m}} \Lambda_{1}\right)$ is right $\Lambda$-projective, the sequence splits when considered as a sequence of right $\Lambda$-modules, and consequently the sequence

$$
0 \rightarrow M \rightarrow K_{\eta} \otimes_{\Lambda} M \rightarrow \Omega_{\Lambda^{\mathrm{e}}}^{t m-1}\left(\psi_{\psi^{m}} \Lambda_{1}\right) \otimes_{\Lambda} M \rightarrow 0
$$

is exact. For each $i \geq 0$ the latter sequence induces a long exact sequence

$$
\begin{gathered}
\operatorname{Ext}_{\Lambda}^{t i}\left(\psi_{\psi^{i}}\left(K_{\eta} \otimes_{\Lambda} M\right), \Lambda / \mathfrak{r}\right) \rightarrow \operatorname{Ext}_{\Lambda}^{t i}\left(\psi^{i} M, \Lambda / \mathfrak{r}\right) \stackrel{\partial_{t i}}{\longrightarrow} \\
\operatorname{Ext}_{\Lambda}^{t(i+m)}\left({ }_{\psi^{i+m}} M, \Lambda / \mathfrak{r}\right) \rightarrow \operatorname{Ext}_{\Lambda}^{t i+1}\left(\psi_{\psi^{i}}\left(K_{\eta} \otimes_{\Lambda} M\right), \Lambda / \mathfrak{r}\right) \rightarrow \\
\operatorname{Ext}_{\Lambda}^{t i+1}\left(_{\psi^{i}} M, \Lambda / \mathfrak{r}\right) \stackrel{\partial_{t i+1}}{\longrightarrow} \operatorname{Ext}_{\Lambda}^{t(i+m)+1}\left(_{\psi^{i+m}} M, \Lambda / \mathfrak{r}\right)
\end{gathered}
$$

in which we have replaced $\operatorname{Ext}_{\Lambda}^{j}\left(\psi_{\psi^{i}}\left(\Omega_{\Lambda^{\mathrm{e}}}^{t m-1}\left(\psi_{\psi^{m}} \Lambda_{1}\right) \otimes_{\Lambda} M\right), \Lambda / \mathfrak{r}\right)$ with $\operatorname{Ext}_{\Lambda}^{j+t m-1}\left({ }_{\psi^{i+m}} M, \Lambda / \mathfrak{r}\right)$, due to the fact that $\psi_{\psi^{i}}\left(\Omega_{\Lambda^{\mathrm{e}}}^{t m-1}\left(\psi_{\psi^{m}} \Lambda_{1}\right) \otimes_{\Lambda} M\right)$ is a $(t m-1)$ th syzygy of $\psi^{i+m} M$. By Mac, Theorem III.9.1] the connecting homomorphism $\partial_{j}$ is then the Yoneda product with the extension $(-1)^{j} \psi^{i}\left(\eta \otimes_{\Lambda} M\right)$, in particular we see that $\partial_{t i}$ is scalar multiplication with $(-1)^{t i} \eta$.

Now consider the connecting homomorphism $\partial_{t i+1}$. Applying the Yoneda relation (†) from Section 3 to $\eta$ and the short exact sequence

$$
\theta: 0 \rightarrow \Omega_{\Lambda}^{1}(M) \rightarrow P_{0} \rightarrow M \rightarrow 0
$$

gives the relation

$$
\left(\eta \otimes_{\Lambda} \Omega_{\Lambda}^{1}(M)\right) \circ\left({ }_{\psi^{m}} \Lambda_{1} \otimes_{\Lambda} \theta\right)=(-1)^{t m}\left(\Lambda \otimes_{\Lambda} \theta\right) \circ\left(\eta \otimes_{\Lambda} M\right),
$$

which we may twist by $\psi^{i}$ to obtain the relation

$$
\psi^{i}\left(\eta \otimes_{\Lambda} \Omega_{\Lambda}^{1}(M)\right) \circ_{\psi^{i+m}} \theta=(-1)^{t m} \psi_{\psi^{i}} \theta \circ \psi_{\psi^{i}}\left(\eta \otimes_{\Lambda} M\right) .
$$

This gives a commutative diagram

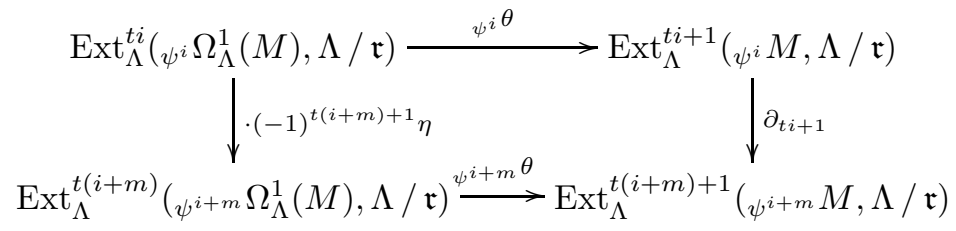

in which the horizontal maps are isomorphisms, hence the connecting homomorphism $\partial_{t i+1}$ is also basically just scalar multiplication with $\eta$, as was the case with $\partial_{t i}$. Consequently they are both injective for $i \gg 0$, giving a short exact sequence

$(\dagger \dagger) 0 \rightarrow{ }_{\Lambda}^{t i}\left(\psi^{i} M, \Lambda / \mathfrak{r}\right) \stackrel{\cdot \eta}{\rightarrow}{ }_{\Lambda}^{t(i+m)}\left(\psi_{\psi^{i+m}} M, \Lambda / \mathfrak{r}\right) \rightarrow{ }_{\Lambda}^{t i+1}\left(\psi_{\psi^{i}}\left(K_{\eta} \otimes_{\Lambda} M\right), \Lambda / \mathfrak{r}\right) \rightarrow 0$

for large $i$ (in which we have used the short hand notion ${ }_{\Lambda}^{j}(-,-$ ) for $\left.\operatorname{Ext}_{\Lambda}^{j}(-,-)\right)$. Note that we may identify $\operatorname{Ext}_{\Lambda}^{t i+1}\left(\psi_{\psi^{i}}\left(K_{\eta} \otimes_{\Lambda} M\right), \Lambda / \mathfrak{r}\right)$ with $\operatorname{Ext}_{\Lambda}^{t i}\left(\psi^{i}\left(\Omega_{\Lambda^{\mathrm{e}}}^{1}\left(K_{\eta}\right) \otimes_{\Lambda} M\right), \Lambda / \mathfrak{r}\right) ;$ since $K_{\eta}$ is right $\Lambda$-projective the projective bimodule cover

$$
0 \rightarrow \Omega_{\Lambda^{\mathrm{e}}}^{1}\left(K_{\eta}\right) \rightarrow Q \rightarrow K_{\eta} \rightarrow 0
$$


of $K_{\eta}$ splits as a sequence of right $\Lambda$-modules, and therefore stays exact when tensored with $M$. In addition, the $\Lambda$-module $Q \otimes_{\Lambda} M$ is projective, hence

$$
\operatorname{Ext}_{\Lambda}^{t i+1}\left(\psi_{\psi^{i}}\left(K_{\eta} \otimes_{\Lambda} M\right), \Lambda / \mathfrak{r}\right) \simeq \operatorname{Ext}_{\Lambda}^{t i}\left(\psi^{i}\left(\Omega_{\Lambda^{\mathrm{e}}}^{1}\left(K_{\eta}\right) \otimes_{\Lambda} M\right), \Lambda / \mathfrak{r}\right) .
$$

Consider now the $H$-module $\operatorname{Ext}_{\Lambda}^{t *}\left(\psi_{\psi^{*}}\left(\Omega_{\Lambda^{\mathrm{e}}}^{1}\left(K_{\eta}\right) \otimes_{\Lambda} M\right), \Lambda / \mathfrak{r}\right)$, and let $w$ be an integer such that the sequence (†t) is exact for $i \geq w$. Then the submodule $\bigoplus_{i=w}^{\infty} \operatorname{Ext}_{\Lambda}^{t i}\left({ }_{\psi^{i}}\left(\Omega_{\Lambda^{\mathrm{e}}}^{1}\left(K_{\eta}\right) \otimes_{\Lambda} M\right), \Lambda / \mathfrak{r}\right)$ is finitely generated over

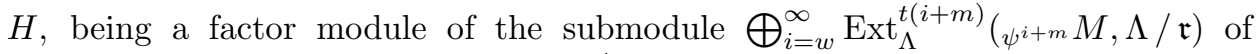
the finitely generated $H$-module $\operatorname{Ext}_{\Lambda}^{t *}\left(\psi^{*} M, \Lambda / \mathfrak{r}\right)$. But then the $H$-module $\operatorname{Ext}_{\Lambda}^{t *}\left(\psi_{\psi^{*}}\left(\Omega_{\Lambda^{\mathrm{e}}}^{1}\left(K_{\eta}\right) \otimes_{\Lambda} M\right), \Lambda / \mathfrak{r}\right)$ must be finitely generated itself, since each graded part $\operatorname{Ext}_{\Lambda}^{t j}\left(\psi_{\psi^{j}}\left(\Omega_{\Lambda^{\mathrm{e}}}^{1}\left(K_{\eta}\right) \otimes_{\Lambda} M\right), \Lambda / \mathfrak{r}\right)$ is finitely generated over $H^{0}$. Also, from Be1, Proposition 3.1] we get

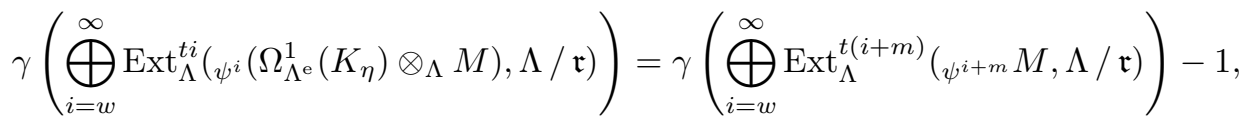

and since for any $\Lambda$-module $X$ the rate of growth of $\operatorname{Ext}_{\Lambda}^{t *}\left(\psi_{\psi^{*}} X, \Lambda / \mathfrak{r}\right)$ equals that of $\bigoplus_{i=w}^{\infty} \operatorname{Ext}_{\Lambda}^{t i}\left(\psi_{\psi^{i}} X, \Lambda / \mathfrak{r}\right)$ we get

$$
\gamma\left(\operatorname{Ext}_{\Lambda}^{t *}\left(\psi^{*}\left(\Omega_{\Lambda^{\mathrm{e}}}^{1}\left(K_{\eta}\right) \otimes_{\Lambda} M\right), \Lambda / \mathfrak{r}\right)\right)=\gamma\left(\operatorname{Ext}_{\Lambda}^{t *}\left(\psi^{*} M, \Lambda / \mathfrak{r}\right)\right)-1
$$

Therefore the equality $\mathrm{cx}^{t}\left(\Omega_{\Lambda^{\mathrm{e}}}^{1}\left(K_{\eta}\right) \otimes_{\Lambda} M\right)=\mathrm{cx}^{t} M-1$ holds, and so from Proposition 4.5 we conclude that $\operatorname{dim} \mathrm{V}_{H}^{\psi}\left(\Omega_{\Lambda^{\mathrm{e}}}^{1}\left(K_{\eta}\right) \otimes_{\Lambda} M\right)=\operatorname{dim} \mathrm{V}_{H}^{\psi}(M)-1$.

Finally we turn to the setting in which $\mathbf{F g}(X, H, \psi, t)$ holds for all $\Lambda$-modules $X$, and derive two corollaries from Proposition 4.8. Observe first that if $\mathbf{F g}(\Lambda / \mathfrak{r}, H, \psi, t)$ holds, then $\mathbf{F g}(S, H, \psi, t)$ holds for every simple $\Lambda$-module $S$, and so by induction on the length of a module we see that $\mathbf{F g}(X, H, \psi, t)$ holds for every $\Lambda$-module $X$; namely, if $\ell(X) \geq 2$, choose a submodule $Y \subset X$ such that $\ell(Y)=\ell(X)-1$. The exact sequence

$$
0 \rightarrow Y \rightarrow X \rightarrow X / Y \rightarrow 0
$$

induces the exact sequence

$$
\operatorname{Ext}_{\Lambda}^{t *}\left(\psi^{*}(X / Y), \Lambda / \mathfrak{r}\right) \rightarrow \operatorname{Ext}_{\Lambda}^{t *}\left(\psi^{*} X, \Lambda / \mathfrak{r}\right) \rightarrow \operatorname{Ext}_{\Lambda}^{t *}\left(\psi_{*}^{*} Y, \Lambda / \mathfrak{r}\right)
$$

of $H$-modules, and since the end terms are finite over $H$, so is the middle term.

Corollary 4.9. If $\mathbf{F g}(\Lambda / \mathfrak{r}, H, \psi, t)$ holds and $\operatorname{dim} \mathrm{V}_{H}^{\psi}(M)=d>0$, then there exist homogeneous elements $\eta_{1}, \ldots, \eta_{d-1} \in H$ of positive degrees such that the module

$$
\Omega_{\Lambda^{\mathrm{e}}}^{1}\left(K_{\eta_{d-1}}\right) \otimes_{\Lambda} \cdots \otimes_{\Lambda} \Omega_{\Lambda^{\mathrm{e}}}^{1}\left(K_{\eta_{1}}\right) \otimes_{\Lambda} M
$$

is eventually $\psi^{i}$-periodic for some $i \geq 1$.

Proof. This is a direct consequence of Proposition 4.6 and Proposition 4.8.

Corollary 4.10. Suppose $k$ is a field and $\Lambda$ is a Frobenius algebra, and let $\Lambda \stackrel{\nu}{\rightarrow} \Lambda$ be a Nakayama automorphism. If $\mathbf{F g}\left(\Lambda / \mathfrak{r}, H, \nu^{n}, 2 n\right)$ holds for some $n \geq 1$ and $\operatorname{dim} \mathrm{V}_{H}^{\nu}(M)=d>0$, then there exist homogeneous elements $\eta_{1}, \ldots, \eta_{d-1} \in H$ of positive degrees such that every nonzero nonprojective indecomposable summand of

$$
\Omega_{\Lambda^{\mathrm{e}}}^{1}\left(K_{\eta_{d-1}}\right) \otimes_{\Lambda} \cdots \otimes_{\Lambda} \Omega_{\Lambda^{\mathrm{e}}}^{1}\left(K_{\eta_{1}}\right) \otimes_{\Lambda} M
$$

is $\tau$-periodic.

Proof. This is a direct consequence of Proposition 4.7 and Proposition 4.8. 


\section{ACKNOWLEDGEMENT}

I would like to thank my supervisor Øyvind Solberg for valuable suggestions and comments on this paper.

\section{REFERENCES}

[Asa] H. Asashiba, The derived equivalence classification of representation-finite self-injective algebras, J. Algebra 214 (1999), 182-221.

[ASS] I. Assem, D. Simson, A. Skowronski, Elements of the representation theory of associative algebras, vol. 1, Cambridge University Press, 2006.

[ARS] M. Auslander, I. Reiten, S. Smalø, Representation theory of Artin algebras, Cambridge University Press (1995).

[Avr] L. Avramov, Modules of finite virtual projective dimension, Invent. Math. 96 (1989), 71-101.

[AvB] L. Avramov, R.-O. Buchweitz, Support varieties and cohomology over complete intersections, Invent. Math. 142 (2000), 285-318.

[Ben] D. Benson, Representations and cohomology, vol. II, Cambridge University Press, 1991.

[Be1] P.A. Bergh, Complexity and periodicity, Colloq. Math. 104 (2006), no. 2, 169-191.

[Be2] P.A. Bergh, On the Hochschild (co)homology of quantum exterior algebras, to appear in Comm. Algebra.

[BGMS] R.-O. Buchweitz, E. Green, D. Madsen, Ø. Solberg, Finite Hochschild cohomology without finite global dimension, Math. Res. Lett. 12 (2005), no. 5-6, 805-816.

[Car] J. Carlson, The varieties and the cohomology ring of a module, J. Algebra 85 (1983), no. $1,104-143$

[CaE] H. Cartan, S. Eilenberg, Homological Algebra, Princeton University Press, 1956.

[EHSST] K. Erdmann, M. Holloway, N. Snashall, Ø. Solberg, R. Taillefer, Support varieties for selfinjective algebras, $K$-theory 33 (2004), 67-87.

[EHS] K. Erdmann, T. Holm, N. Snashall, Twisted bimodules and Hochschild cohomology for self-injective algebras of class $A_{n}$, II, Algebr. Represent. Theory 5 (2002), no. 5, 457-482.

[JoS] D. Jorgensen, L. Sega, Nonvanishing cohomology and classes of gorenstein rings, Adv. Math. 188 (2004), no. 2, 470-490.

[LiS] S. Liu, R. Schulz, The existence of bounded infinite D Tr-orbits, Proc. Amer. Math. Soc. 122 (1994), no. 4, 1003-1005.

[Mac] S. Mac Lane, Homology, Classics in Mathematics, Springer-Verlag, 1995.

[M-H] F. Membrillo-Hernández, Homological properties of finite dimensional algebras, DPhil thesis, University of Oxford, 1993.

[Po1] Z. Pogorzały, Invariance of Hochschild cohomology algebras under stable equivalences of Morita type, J. Math. Soc. Japan 53 (2001), 913-918.

[Po2] Z. Pogorzaly, A new invariant of stable equivalences of Morita type, Proc. Amer. Math. Soc. 131 (2003), no. 2, 343-349.

[Po3] Z. Pogorzały, On the Auslander-Reiten periodicity of self-injective algebras, Bull. London Math. Soc. 36 (2004), 156-168.

[Po4] Z. Pogorzały, Auslander-Reiten orbit algebras for self-injective Nakayama algebras, Algebra Colloq. 12 (2005), no. 2, 351-360.

[Rie] C. Riedtmann, Representation-finite self-injective algebras of class $A_{n}$, in Representation theory II, Proc. Second Internat. Conf., Carleton Univ., Ottawa, Lecture Notes in Math. 832, Springer, 1980, 449-520.

[Rot] J. Rotman, An introduction to homological algebra, Academic Press, 1979.

$[\mathrm{ScW}] \quad$ E. Scherzler, J. Waschbüsch, A class of self-injective algebras of finite representation type, in Representation theory II, Proc. Second Internat. Conf., Carleton Univ., Ottawa, Lecture Notes in Math. 832, Springer, 1980, 545-572.

[Sch] R. Schulz, Boundedness and periodicity of modules over QF rings, J. Algebra 101 (1986), no. $2,450-469$.

[Sma] S. Smalø, Local limitations of the Ext functor do not exist, Bull. London Math. Soc. 38 (2006), no. 1, 97-98.

[SnS] N. Snashall, Ø. Solberg, Support varieties and Hochschild cohomology rings, Proc. London Math. Soc. 88 (2004), 705-732.

[Yon] N. Yoneda, Note on products in Ext, Proc. Amer. Math. Soc. 9 (1958), 873-875.

Petter Andreas Bergh

Institutt For MATEMATISke FAG, NTNU, N-7491 Trondheim, NorWAY

E-mail address: bergh@math.ntnu.no 